\title{
FACTOR DE COMPORTAMIENTO SÍSMICO MÁXIMO DE DISEÑO PARA EDIFICIOS UBICADOS EN EL MUNICIPIO DE VERACRUZ, Y DISEÑADOS CON EL MDOC-CFE-2015
}

\author{
José E. Barradas Hernández ${ }^{(1)}$, Alejandro Vargas Colorado ${ }^{(1)}$ y \\ Yasser Picazo Gama ${ }^{(2)}$
}

\begin{abstract}
RESUMEN
En este estudio se muestra que diseñar un edificio para valores del factor de comportamiento sísmico $(Q)$ de tres o cuatro, de acuerdo con el Manual de Diseño de Obras Civiles de la Comisión Federal de Electricidad versión 2015 (MDOC-CFE-2015), podría resultar en que el estado límite de servicio rija el diseño por resistencia de una estructura, lo cual no debe ser. Para percatarse de ello, el diseñador tendrá que realizar un proceso iterativo que podría requerir muchas iteraciones, lo cual incrementará significativamente el trabajo. Por lo anterior, se obtiene una función para estimar el máximo valor de $Q$ con el que se debe diseñar un edificio, cuya implementación reduce considerablemente dicho trabajo iterativo.
\end{abstract}

Palabras clave: Factor de comportamiento sísmico; diseño sísmico; manual de diseño de obras civiles

\section{MAXIMUM DESIGN SEISMIC BEHAVIOR FACTOR FOR BUILDINGS LOCATED IN THE CITY OF VERACRUZ, AND DESIGNED WITH THE MDOC-CFE-2015}

\begin{abstract}
This study shows that in the process of the seismic design of a building for values of the seismic behavior factor $(Q)$ of three or four, according to the Manual of Civil Works Design of the Federal Electricity Commission (MDOC-CFE-2015, for its acronym in Spanish), it may occur that the serviceability limit state governs the strength design of a structure, which it should not be. Thus, the designer will have to go through an iterative process that could require many iterations, significantly increasing the work. Therefore, a function is obtained to estimate the maximum value of $\mathrm{Q}$ to design a building, whose implementation considerably reduces said iterations.
\end{abstract}

Keywords: Seismic behaviour factor; seismic design; Civil Works Design Handbook of the CFE 2015

Artículo recibido el 27 de febrero de 2020 y aprobado para su publicación el 29 de marzo de 2021. Se aceptarán comentarios y/o discusiones hasta cinco meses después de su publicación.

(1) Instituto de Ingeniería, Universidad Veracruzana, Av. S.S. Juan Pablo II s/n, Costa Verde, Boca del Río, Veracruz, C.P. 94294. erbarradas@uv.mx ; alejvargas@uv.mx

(2) Instituto Nacional de Electricidad y Energías Limpias, Reforma 113, Palmira Tinguindin, Cuernavaca, Morelos, C.P. 62490. yasserpicazo@yahoo.com.mx

DOI: $10.18867 /$ ris. 105.566 


\section{INTRODUCCIÓN}

Un objetivo de las recomendaciones del Capítulo de Diseño por Sismo del Manual de Diseño de Obras Civiles versión 2015 de la Comisión Federal de Electricidad (CDS-MDOC-2015), (CFE, 2015) es: "Fijar los requisitos mínimos para el diseño sísmico de estructuras y obras civiles para que sean capaces de resistir: (a) sismos de poca intensidad sin daño, (b) sismos moderados sin daño estructural, pero posiblemente con algún daño a los elementos no estructurales, y (c) un sismo fuerte con daños a elementos estructurales y no estructurales, sin llegar al colapso." Al estado de una estructura dado por el grado de daño que exhiben sus elementos estructurales y no estructurales en el primer y tercer criterio de desempeño, se les denomina estado límite de servicio (ELS) y estado límite de prevención de colapso (ELPC) respectivamente.

Estructuras convencionales para uso comercial, habitacional y de oficinas son muy comunes en las medianas y grandes ciudades. De acuerdo con el CDS-MDOC-2015 las estructuras anteriores y algunas para otros usos, pueden clasificarse dentro del denominado grupo B. El diseño de estas estructuras requiere un grado de seguridad convencional debido a que su falla ocasionaría pérdidas económicas moderadas. Además, un porcentaje significativo de estas estructuras tienen las características (altura mayor que 13 metros o área total construida mayor que $400 \mathrm{~m}^{2}$ ) para clasificarlas dentro de un subgrupo denominado clase 1. En el CDS-MDOC-2015 a estructuras del grupo B, clase 1, suele denominárseles de forma abreviada estructuras con importancia estructural B1.

En el CDS-MDOC-2015 se indica que para realizar la revisión de deformaciones laterales (dimensionamiento por rigidez lateral) en el ELPC de estructuras con importancia estructural B1, se debe utilizar el denominado espectro de diseño modificado o reducido (EDM). Con este espectro también se debe obtener el cortante basal elástico de diseño (Vb-diseño) de una estructura. El EDM se obtiene dividiendo cada una de las ordenadas del denominado espectro de diseño transparente regional, por el siguiente producto de constantes y funciones: $\alpha Q^{\prime}\left(T_{e}, Q\right) R\left(T_{e}, R_{o}\right) \rho$, que en el presente trabajo se denomina factor reductor total $(F R T)$. Donde $\alpha$ es una constante denominada factor correctivo por irregularidad; $Q$ ' es el factor reductor por ductilidad, el cual es función del periodo estructural $\left(T_{e}\right)$ y del factor de comportamiento sísmico, $Q ; R$ es el factor reductor por sobrerresistencia, el cual es función de $T_{e}$ y de la denominada sobrerresistencia índice, $R_{o}$; y $\rho$ es una constante denominada factor por redundancia. De acuerdo con las recomendaciones del CDS-MDOC-2015, las ordenadas del espectro de diseño transparente regional representan únicamente el peligro sísmico esperado en estructuras del grupo B, y no están reducidas por algún factor ajeno a dicho peligro; por lo tanto, las ordenadas de este espectro son elásticas.

En el CDS-MDOC-2015 se especifica que para realizar el dimensionamiento por rigidez lateral en el ELS de estructuras tipo edificio del grupo B, se debe emplear el denominado espectro de diseño para el ELS (ED-ELS). Este espectro se obtiene reduciendo cada una de las ordenadas del espectro de diseño transparente regional, únicamente por el denominado factor de servicio, $F_{\text {ser }}$, el cual tiene un valor constante igual a 5.5; sin reducciones por ductilidad, sobrerresistencia ni redundancia. Esto implica que las ordenadas de este espectro son completamente elásticas, y por lo tanto la respuesta estructural ante el sismo de diseño correspondiente al ELS también debe ser completamente elástica.

Para garantizar que la respuesta de una estructura tipo edificio ante el sismo de diseño asociado al ELS sea elástica, se requiere que las ordenadas del EDM correspondientes al menos a sus periodos fundamentales en ambas direcciones traslacionales sean mayores que las correspondientes ordenadas en el ED-ELS. El CDS-MDOC-2015, como otras normas de diseño sísmico del país, contempla que las estructuras tipo edificio pueden diseñarse para valores de $Q$ hasta de cuatro. En el presente trabajo se muestra que, si una estructura tipo edificio cuyos periodos fundamentales traslacionales se ubican después de la rama ascendente de los espectros de diseño, se diseña siguiendo los lineamientos del CDS-MDOC-2015 para un 
valor de $Q=4$; las ordenadas correspondientes a dichos periodos en el EDM serán menores que las correspondientes ordenadas en el ED-ELS. Este resultado indicaría que el ELS rige el diseño por resistencia de la estructura, lo cual no debe ser. Para percatarse de ello, el diseñador tendrá que realizar un proceso iterativo que podría requerir muchas iteraciones, lo cual incrementará significativamente el trabajo. En el presente artículo se obtiene una función para estimar el máximo valor de Q con el que se debe diseñar un edificio, con cuya implementación se reduce considerablemente dicho trabajo iterativo y se cumplen simultáneamente los criterios de desempeño para ambos estados límite.

\section{DEFINICIÓN DE LOS PARÁMETROS DE DISEÑO SÍSMICO}

La fig. 1, adaptada de Uang, (1991), muestra para una estructura tipo edifico que posea cierta capacidad dúctil de deformación lateral y sea capaz de disipar energía de manera estable, sus curvas de respuesta ante carga lateral, real e idealizada como bilineal; definidas por la relación entre su fuerza cortante basal $(V b)$ y el desplazamiento lateral de su extremo superior $(4)$. La curva bilineal define la relación fuerzadeformación lateral idealizada de la estructura, a partir de la cual se definen los parámetros $Q^{\prime}, Q, R$ y $\rho$ para su diseño sísmico. Cuando la curva de respuesta real no presenta un decremento de pendiente brusco que indique claramente la fluencia de la estructura, la curva bilineal suele idealizarse como elastoplástica perfecta (Elnashai y Mwafy, 2002).

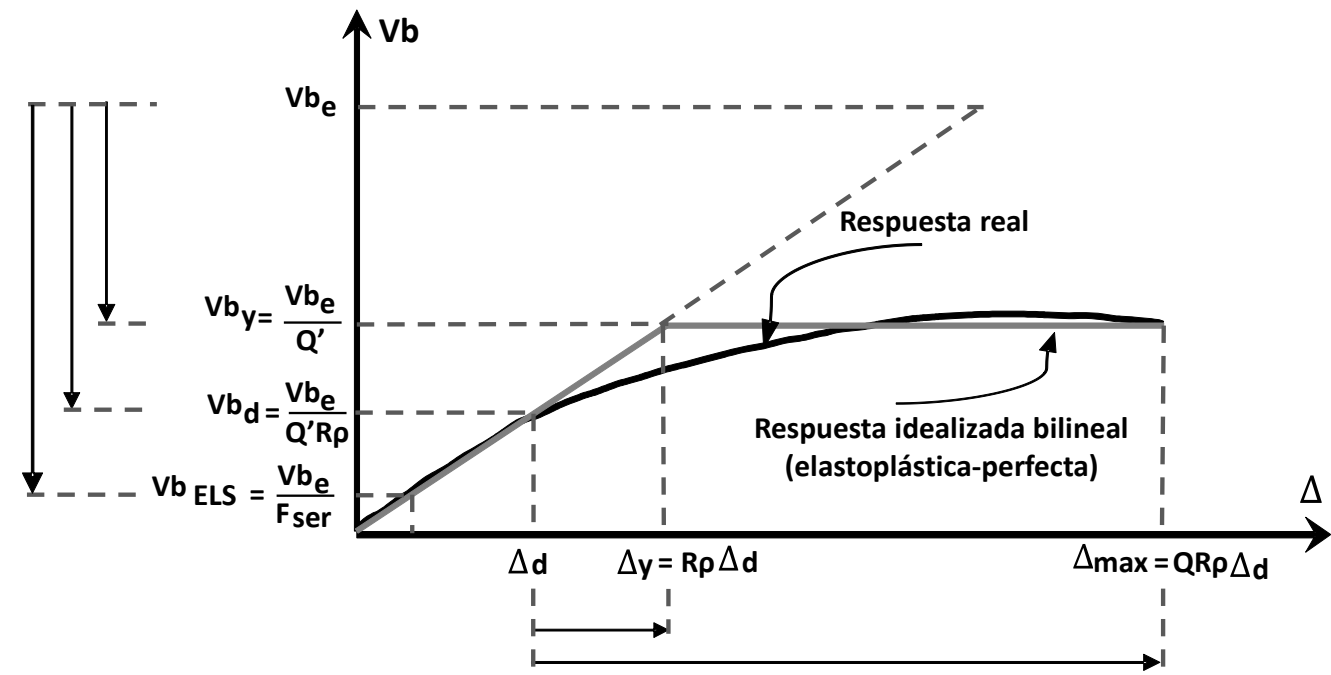

Figura 1. Relación fuerza cortante basal - desplazamiento lateral del extremo superior de una estructura.

La rama inicial de la curva idealizada es una recta secante que parte del origen e intersecta a la curva de respuesta real en un punto cuya ordenada es el $\mathrm{Vb}$-diseño $\left(V b_{d}\right)$. El desplazamiento lateral del extremo superior de la estructura correspondiente al Vb-diseño es $\Delta_{d}$. El factor $Q$ ' se define mediante la ec. 1

$Q^{\prime}=\frac{V b_{e}}{V b_{y}}$

donde $V b_{e}$ es el cortante basal requerido para que la respuesta de la estructura sea elástica ante el sismo de diseño correspondiente al ELPC, y $V b_{y}$ es el cortante basal para la cual, de forma idealizada, se inicia la fluencia significativa de la estructura. Como se muestra en la fig. 1, $V b_{y}$ es la ordenada del punto de cambio de pendiente de la curva bilineal idealizada y la correspondiente abscisa es $\Delta_{y}$. En el CDS-MDOC-2015 se 
menciona que los factores $R$ y $\rho$ están relacionados por la hiperestaticidad de una estructura, por lo que su producto, que se puede interpretar como su sobrerresistencia total, se define mediante la ec.2:

$R \rho=\frac{V b_{y}}{V b_{d}}=\frac{\Delta_{y}}{\Delta_{d}}$

El factor $Q$ es una medida de la capacidad dúctil global de deformación lateral de una estructura, definida como la relación de la capacidad de desplazamiento lateral de una estructura $\left(\Delta_{\max }\right)$ al desplazamiento de su inicio de fluencia significativa, $\Delta_{y}$. De acuerdo con lo anterior, $Q$ se puede describir mediante la ec. 3:

$Q=\frac{\Delta_{\max }}{\Delta_{y}}=\frac{\Delta_{\max }}{R \rho \Delta_{d}}$

Combinando las ecuaciones anteriores es posible describir el Vb-diseño mediante la ec. 4:

$V b_{d}=\frac{V b_{e}}{Q^{\prime} R \rho}$

Además, el cortante basal correspondiente al ELS (Vb-ELS, $V b_{E L S}$ ) se puede describir mediante la ec. 5:

$V b_{E L S}=\frac{V b_{e}}{F_{\text {ser }}}$

\section{IDENTIFICACIÓN DEL ESTADO LÍMITE QUE RIGE EL DISEÑO POR RESISTENCIA}

En la sección 3.3.7.1 de los Comentarios del CDS-MDOC-2015 titulada "Desplazamientos horizontales por limitación de daños a elementos no estructurales (límite de servicio)", se menciona lo siguiente: "se establecen límites en los desplazamientos obtenidos para el nivel de servicio porque se debe evitar daño en elementos no estructurales en sismos menores que el considerado para la resistencia de la estructura ante el nivel de colapso, en que se debe garantizar que la estructura mantenga su estabilidad". Si se tiene en cuenta que el EDM representa el sismo considerado para la resistencia de la estructura ante el nivel de colapso y que el ED-ELS representa los sismos menores, al parecer, en el CDS-MDOC-2015 se considera que para todo $T_{e}$ las ordenadas del EDM serán mayores que las del ED-ELS. Sin embargo, como se muestra a continuación, esto depende del valor del FRT obtenido en el diseño.

Considérese que se realizará el diseño sísmico de tres edificios (Edificio 1, 2 y 3) con importancia estructural B1 ubicados en un sitio del municipio de Veracruz, que tienen diferentes sistemas estructurales, características geométricas, grados de redundancia estructural, así como potenciales sobrerresistencia y capacidad dúctil de deformación lateral; y por lo tanto se deben diseñar para diferentes valores de los parámetros de diseño sísmico $\alpha, \rho, Q y R_{o}$. Para esto, primero se calculó el espectro de diseño transparente regional en el sitio referido (considerando el valor implícito del amortiguamiento estructural, $\zeta_{\mathrm{e}}=5 \%$ ), cuyo terreno se clasificó hipotéticamente como tipo II de acuerdo al CDS-MDOC-2015. A partir de dicho espectro se calculó el ED-ELS (puesto que el $F_{\text {ser }}$ aplica para ambas direcciones traslacionales de análisis) y para cada edificio sus respectivos EDM (EDM 1, 2 y 3). Para el cálculo de los EDM se ignoró la interacción suelo-estructura y se consideró que sus respectivos edificios cumplen con el conjunto de requisitos necesarios en ambas direcciones traslacionales, para emplear los siguientes valores de los parámetros de diseño sísmico: EDM-1: $\alpha=1, Q=2, R_{o}=2, \rho=1.25$; EDM-2: $\alpha=1, Q=3, R_{o}=2, \rho=1.25$ у EDM3: $\alpha=1, Q=4, R_{o}=3, \rho=1.25$. En la fig. 2 se muestran los cinco espectros calculados. 


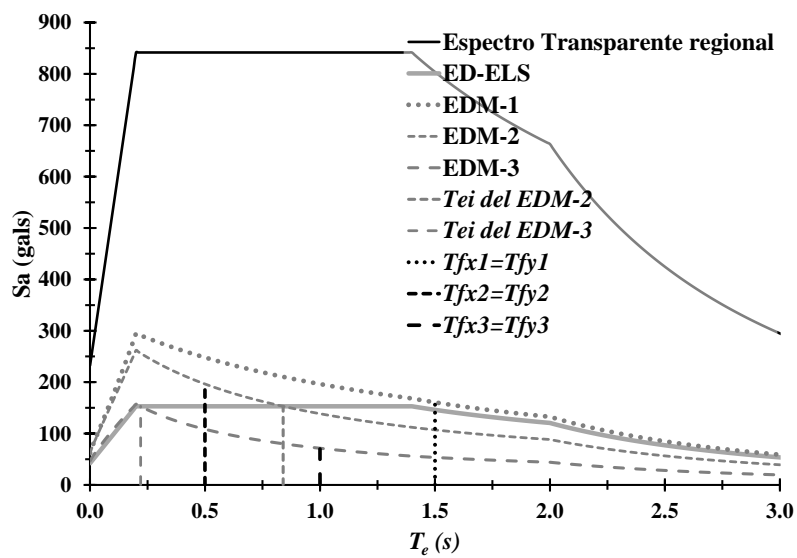

Figura 2. Espectros de diseño para un sitio del municipio de Veracruz con terreno tipo II en la región sísmica B

En la fig. 2 se observa que los EDM 2 y 3 se intersecan con el ED-ELS, y que antes y después de dicha intersección las ordenadas de ambos EDM son mayores y menores, respectivamente, a las correspondientes ordenadas del ED-ELS. En el presente trabajo a las abscisas de los puntos de intersección de los espectros se les denomina periodo estructural de intersección $\left(T_{e i}\right)$. Los $T_{e i}$ de los EDM 2 y 3 con el ED-ELS son $0.84 \mathrm{~s}$ y $0.21 \mathrm{~s}$, respectivamente. El que las ordenadas en los EDM 2 y 3 correspondientes a periodos estructurales mayores que los respectivos $T_{e i}$ en dichos espectros, sean menores que su correspondiente ordenada en el ED-ELS; se debe a que para tales periodos estructurales el valor del FRT es mayor que el $F_{\text {ser }}$, como se muestra en la fig. 3. En dicha figura también se muestran los periodos de inicio y término de la meseta del espectro de diseño transparente regional, $T_{a}$ y $T_{b}$, y la gráfica del $F R T$ correspondiente al EDM-1; cuyos valores en todo el intervalo de periodos estructurales son menores que el $F_{\text {ser. }}$.

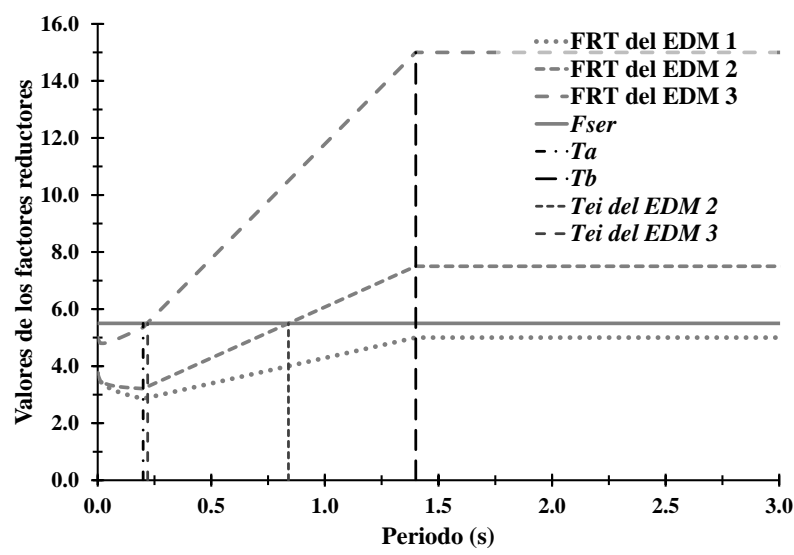

Figura 3. Factores reductores correspondientes a los espectros de diseño

Dado el valor del $F_{s e r}$, la ubicación del $T_{e i}$ en un EDM depende de la combinación de valores de los parámetros de diseño sísmico $\alpha, \rho, Q$ y $R_{o}$ aplicables a la estructura. De estos parámetros, los que menor influencia tienen en las ordenadas del EDM son $\alpha$ y $\rho$ debido a que los valores que pueden tomar son cercanos a la unidad: $(0.7,0.8,0.9$ y 1.0$)$ para $\alpha$ y $(0.8,1.0$ y 1.25$)$ para $\rho$. Los que mayor influencia tienen son $R_{o}$ y $Q$ debido a que los valores que pueden tomar son de moderada a significativamente mayores que la unidad y porque además, entre mayor sea el valor de $Q$ que aplique, mayor será el correspondiente valor aplicable de $R_{o}$. El valor mínimo del $T_{e i}$ se presenta en el EDM cuyas ordenadas son las mínimas posibles, 
las cuales corresponden a la combinación de los valores máximos de los parámetros de diseño sísmico: $\alpha=1.0, \rho=1.25$. $Q=4$, y $R_{o}=3$. De los espectros mostrados en la fig. 2, el EDM-3 corresponde a estos valores de los parámetros de diseño sísmico, por lo que al menos para espectros regionales correspondientes a suelo tipo II en la región sísmica B, el $T_{e i}$ mínimo no se ubica en la rama ascendente de sus EDM. Los autores del presente trabajo han observado resultados similares a los previamente discutidos en esta sección, considerando espectros regionales para suelos tipo I y III, todos en la región sísmica B. No se han investigado estos aspectos en los espectros regionales para los tres tipos de suelo de las restantes regiones sísmicas del país. Sin embargo, considerando que los siguientes aspectos para construir los espectros regionales en todas las regiones sísmicas y sus correspondientes EDM y ED-ELS, son iguales: (1) las ecuaciones que definen a las diferentes ramas de los espectros regionales transparentes, (2) las funciones 0 constantes que definen a los factores reductores $Q^{\prime}\left(Q, T_{e}\right), R\left(R_{o}, T_{e}\right)$ y $F_{s e r} \mathrm{y}(3)$, los valores de los parámetros de diseño sísmico $\alpha, \rho Q, y R_{o}$; se piensa que se obtendrán resultados similares.

En la fig. 2 se observó que los EDM 2 y 3 se intersecan con el ED-ELS, y que antes y después de dicha intersección las ordenadas de ambos EDM son mayores y menores, respectivamente, a las correspondientes ordenadas del ED-ELS. En estructuras tipo edificio la contribución de sus modos fundamentales traslacionales a su respuesta total comúnmente es muy significativa, mientras que la contribución de sus modos superiores suele ser pequeña o moderada. Considerando lo anterior, si al realizar el dimensionamiento por rigidez lateral de los edificios 2 y 3, las ordenadas en los EDM 2 y 3 correspondientes a sus periodos fundamentales traslaciones son menores que las correspondientes ordenadas en el ED-ELS, es posible que el Vb-diseño en ambas direcciones de estos edificios sean menores que sus correspondientes cortantes basales en el ELS, Vbs-ELS; ya que la mayor contribución a los cortantes basales en ambos estados límite se debe a los modos fundamentales traslacionales. Si las ordenadas en los EDM 2 y 3 correspondientes a los periodos superiores traslaciones de la estructura son mayores que las correspondientes ordenadas en el ED-ELS; la contribución de los modos superiores al Vb-diseño será mayor que su contribución al Vb-ELS. Sin embargo, no se espera que la aportación de esta contribución sea la suficiente como para lograr que los Vb-diseño en ambas direcciones sean mayores que los correspondientes Vb-ELS.

Si una estructura se sujeta a un sismo que la induzca a ingresar significativamente dentro su intervalo de comportamiento inelástico, como se muestra en la fig. 1, durante la transición gradual de su respuesta elástica a su respuesta inelástica, primero se desarrolla la fracción elástica de la sobrerresistencia de la estructura, posteriormente su fracción inelástica y finalmente su ductilidad. El escenario en el que el Vbdiseño de una estructura es menor que su Vb-ELS estrictamente no es aceptable, ya que para que la estructura alcance el Vb-ELS debe desarrollar al menos cierta fracción de su sobrerresistencia e incluso de su ductilidad, y como se mencionó, el ED-ELS no está reducido por sobrerresistencia ni por ductilidad; por lo que una estructura no debe desarrollar incluso ni la fracción elástica de su sobrerresistencia ante el sismo de diseño correspondiente al ELS. Si el Vb-diseño de una estructura es solo ligeramente menor que el VbELS, es posible que cuando se someta al sismo correspondiente al ELS su respuesta no exceda la respuesta correspondiente a la fracción elástica de la sobrerresistencia y su comportamiento en el ELS sea elástico, como se requiere. Sin embargo, si el Vb-diseño de una estructura es de moderada a significativamente menor que su Vb-ELS, cuando se sujete al sismo correspondiente al ELS puede desarrollar la suficiente sobrerresistencia e incluso ductilidad, como para que su respuesta sea inelástica.

Si la respuesta de una estructura ante el sismo de diseño correspondiente al ELS es inelástica, cierto número de miembros estructurales desarrollarán demandas de deformación plástica (daño estructural), por lo que necesitarán ser rehabilitados. Al realizar los trabajos de rehabilitación se interrumpirá parcial o incluso totalmente el servicio que presta la estructura y no se cumplirá con este criterio de desempeño requerido en el ELS. Más aún, si los miembros estructurales exhiben daño, la rigidez lateral de la estructura será menor que la estimada en el diseño y consecuentemente sus deformaciones laterales serán mayores que 
las estimadas en el diseño, por lo que los elementos no estructurales exhibirán daño y tampoco se cumplirá con este criterio de desempeño requerido en el ELS. Estos resultados indicarían que el ELS rige el diseño por resistencia de la estructura, lo cual no debe ser. Para percatarse de ello, el diseñador tendrá que realizar un proceso iterativo que podría requerir muchas iteraciones, lo cual incrementará significativamente el trabajo. Por lo anterior es deseable estimar el máximo valor de Q con el que se debe diseñar un edificio, con cuya implementación se reduzca considerablemente dicho trabajo iterativo y se cumplen simultáneamente los criterios de desempeño para ambos estados límite.

\section{PLANTEAMIENTO PARA OBTENER EL FACTOR Q MÁXIMO CON EL QUE SE CUMPLE SIMULTÁNEAMENTE CON AMBOS ESTADOS LÍMITE}

Antes de iniciar los análisis sísmicos se recomienda obtener una gráfica similar a la fig. 2 en la que se muestren los EDM en ambas direcciones traslacionales de análisis, el ED-ELS y valores estimados de los periodos fundamentales traslacionales de la estructura a diseñar. Los EDM se deben calcular para los valores de $\alpha, Q, R_{o}$ y $\rho$ aplicables a la estructura en la correspondiente dirección de análisis. Para estimar los valores de los periodos fundamentales de la estructura se puede usar alguna fórmula aproximada de las disponibles en la literatura especializada, que se considere adecuada de acuerdo al tipo de sistema estructural. En la gráfica obtenida se pueden presentar los siguientes tres escenarios:

1. Si en la gráfica se observa que para todo $T_{e}$ las ordenadas de ambos EDM son mayores que las ordenadas del ED-ELS (los espectros no se intersecan), al diseñarse la estructura con estos espectros sus $\mathrm{Vb}$-diseño en ambas direcciones serán mayores que los correspondientes $\mathrm{Vb}$-ELS, que es el escenario deseable. Con referencia a la fig. 2 este escenario está representado mediante el EDELS, el EDM-1 y los periodos Tf $x l=T f y 1$.

2. Si en la gráfica se observa que un EDM (o ambos) en cierta dirección se intersecta con el ED-ELS, y que la ordenada en dicho EDM correspondiente al periodo fundamental estimado en esa dirección (o ambas direcciones) es mayor a la correspondiente ordenada en el ED-ELS; es posible que al diseñarse la estructura con estos espectros su Vb-diseño en dicha dirección (o ambas direcciones) sea mayor que el correspondiente Vb-ELS, que es el escenario deseable. Con referencia a la fig. 2, el escenario de que ambos EDM se intersecan con el ED-ELS y que las ordenadas en ambos EDM correspondientes a los periodos fundamentales estimados en ambas direcciones son mayores que las correspondientes ordenadas en el ED-ELS; está representado mediante el ED-ELS, el EDM-2 y los periodos $T f x 2=T f y 2$.

3. Si en la gráfica se observa que un EDM (o ambos) en cierta dirección se intersecta con el ED-ELS, y que la ordenada en dicho EDM correspondiente al periodo fundamental estimado en esa dirección (o ambas direcciones) es menor a la correspondiente ordenada en el ED-ELS; es posible que al diseñarse la estructura con estos espectros su Vb-diseño en dicha dirección (o ambas direcciones) sea menor que el correspondiente Vb-ELS. Con referencia a la fig. 2, el escenario de que ambos EDM se intersecan con el ED-ELS y que las ordenadas en ambos EDM correspondientes a los periodos fundamentales estimados en ambas direcciones son menores que las correspondientes ordenadas en el ED-ELS; está representado mediante el ED-ELS, el EDM-3 y los periodos $T f x 3=T f y 3$.

Es importante tener en cuenta que las inferencias obtenidas en los dos últimos escenarios solo son indicativas, ya que los valores requeridos de los periodos fundamentales en ambas direcciones traslacionales y los cortantes basales de ambos estados límite solo pueden conocerse con certeza al realizar el diseño de la estructura. 
Si la implementación del procedimiento anterior proporcionó indicios de que al realizar el diseño de la estructura considerando los EDM calculados con los valores de $\alpha, Q, R_{o}$ y $\rho$ aplicables, es posible que el $\mathrm{Vb}$-diseño en una o ambas direcciones sea menor que el correspondiente $\mathrm{Vb}$-ELS, se proponen dos soluciones: una práctica y otra económica. La solución práctica consiste en diseñar la estructura para un valor discreto de $Q$ y su correspondiente valor discreto de $R_{o}$, especificados en el CDS-MDOC-2015, lo suficientemente menores que los inicialmente aplicables; de tal forma que para todo $T_{e}$ las ordenadas de ambos EDM sean en la medida de lo posible, ligeramente mayores que las del ED-ELS. Dicho valor de $Q$ y su correspondiente $R_{o}$ pueden obtenerse por tanteos.

La solución económica consiste en diseñar la estructura con una función del $F R T$ cuyos valores en el intervalo completo de periodos estructurales sean menores o a lo más iguales que el valor del $F_{\text {ser }}$, de tal forma que las ordenadas de los correspondientes EDM sean mayores o a lo menos iguales que las ordenadas del ED-ELS. Como se mencionó, $F R T=\alpha Q^{\prime}\left(T_{e}, Q\right) R\left(T_{e}, R_{o}\right) \rho$. Para lograr que los valores obtenidos con dicha función sean menores, se considera adecuado diseñar la estructura para una demanda de ductilidad menor que su capacidad dúctil global de deformación lateral, $Q$, prescrita para la cual puede diseñarse de acuerdo con el CDS-MDOC-2015. Considerando con lo anterior, se propone obtener el valor máximo del factor de comportamiento sísmico $\left(Q_{\max }\right)$ con el cual los valores de la función $F R T$ en el intervalo completo de periodos estructurales sean menores o a lo más iguales que el valor del $F_{s e r}$. Por lo anterior, $Q_{\max }$ se puede interpretar como la demanda de ductilidad global correspondiente a la resistencia lateral requerida por el sismo de diseño correspondiente al ELS.

A continuación se describe un planteamiento para obtener el valor de $Q_{\max }$, que se puede implementar en espectros regionales para los tres tipos de terreno en todas las regiones sísmicas del país. Sin embargo, en el presente trabajo se harán las siguientes consideraciones para simplificar su obtención e implementarlo en espectros regionales para terreno tipo II en la región sísmica B: (1) en la sección anterior del presente documento se mostró que al menos para espectros regionales correspondientes a terreno tipo II en la región sísmica B, el $T_{e i}$ no se ubica en la rama ascendente de los EDM, por lo que se obtendrán valores de $Q_{\max }$ solo para las restantes ramas espectrales; (2) teniendo en cuenta que la función Q'( $\left.T_{e}, \mathrm{Q}\right)$ especificada para la meseta es diferente a la especificada para ambas ramas descendentes, solo se deben obtener un par de valores de $Q_{\max }$; (3) de acuerdo con el CDS-MDOC-2015, $R=R_{o}$ si $T_{e}$ es mayor que $T_{a}$, donde $T_{a}$ es el periodo en el que inicia la meseta de los espectros regionales de diseño; (4) aunque en el presente trabajo se está considerando que las estructuras están ubicadas en terreno tipo II, también se considera que dichas estructuras no son susceptibles de desarrollar comportamiento histerético con degradación de rigidez y/o de resistencia, por lo que no aplica el denominado factor de amplificación por comportamiento degradante, $A_{c d}$; (5) no se considera la interacción suelo-estructura. De acuerdo con las consideraciones anteriores, en las ecs. 6 y 7 se plantean las igualdades para obtener $Q_{\max }$ en la meseta y en ambas ramas descendentes, respectivamente:

$\frac{S a_{\text {transparente }}}{F_{\text {ser }}}=\frac{S a_{\text {transparente }}}{\alpha\left[1+(Q-1) \sqrt{\frac{\beta}{k}} \frac{T_{e}}{T_{b}}\right] R_{O} \rho}$
$\frac{S a_{\text {transparente }}}{F_{\text {ser }}}=\frac{S a_{\text {transparente }}}{\alpha\left[1+(Q-1) \sqrt{\frac{\beta P_{b}}{k}}\right] R_{O} \rho}$

donde:

$S a_{\text {transparente }}=$ son las ordenadas del espectro de diseño transparente regional,

$\beta$ es el denominado factor de amortiguamiento, el cual es función de $T_{e} \mathrm{y}$ del amortiguamiento estructural, $\zeta_{e}$,

$k$ es un parámetro que controla la caída de las ordenadas espectrales, 
$T_{b}$ es el periodo que define el límite superior de la meseta del espectro de diseño regional y, $P_{b}$ es un factor empleado para definir la variación del espectro en la rama descendente.

En las igualdades anteriores los términos de la izquierda representan las ordenadas del ED-ELS y los términos de la derecha las ordenadas del EDM. En dichas igualdades se estableció $Q=Q_{\max }$ y se realizaron las operaciones algebraicas necesarias para obtener su valor con el cual se satisfacen cada una de las igualdades, lo que resultó en las ecs. 8 y 9, que son las funciones que definen a $Q_{\max }$ en la meseta y en ambas ramas descendentes, respectivamente:

$Q_{\max }=\frac{\left(F_{\text {ser }}-\alpha R_{O} \rho\right) \sqrt{k} T_{b}+\alpha R_{o} \rho \sqrt{\beta} T_{e}}{\alpha R_{o} \rho \sqrt{\beta} T_{e}}$

$Q_{\max }=\frac{\left(F_{\text {ser }}-\alpha R_{o} \rho\right) \sqrt{k}+\alpha R_{o} \rho \sqrt{\beta} \sqrt{P_{b}}}{\alpha R_{o} \rho \sqrt{\beta} \sqrt{P_{b}}}$

El valor de $Q_{\max }$ obtenido con estas funciones será, en general, diferente a los valores discretos de $Q$ especificados en el CDS-MDOC-2015, y su implementación ayudará al diseñador a cumplir simultáneamente los criterios de desempeño para ambos estados límite. Los EDM se deben construir en dos segmentos. El primer segmento corresponde a valores de $T_{e}$ menores o iguales que el $T_{e i}$ identificado gráficamente, y en las funciones $Q^{\prime}\left(T_{e}, Q\right)$ y $R\left(T_{e}, R_{o}\right)$ que forman parte del $F R T$ se deben usar los valores de $Q$ y su respectivo $R_{o}$, aplicables a la estructura en la correspondiente dirección traslacional. El segundo segmento corresponde a valores de $T_{e}$ mayores que $T_{e i}$, y únicamente en la función $Q^{\prime}\left(T_{e}, Q\right)$ se debe sustituir el valor constante de $Q$ por las funciones que definen a $Q_{\max }$.

Por otra parte, en el CDS-MDOC-2015 se indica que para obtener los desplazamientos laterales para seguridad contra colapso de una estructura en el ELPC, los desplazamientos laterales elásticos obtenidos del análisis con el EDM se deben multiplicar por el producto $Q R\left(T f, R_{o}\right) \rho$, donde $T f$ es el periodo fundamental de la estructura en la dirección traslacional analizada; con lo que se indica que el valor de $R$ que se debe tomar es el correspondiente a dicho periodo. En el presente trabajo al producto anterior se le denomina factor de amplificación de desplazamientos $(F A D)$, y para las direcciones traslacionales de análisis $X$ y $Y$ se definen como: $F A D-X=Q R(T f x, R o) \rho$ y $F A D-Y=Q R(T f y, R o) \rho$, respectivamente, donde $T f x$ y $T f y$ son los periodos fundamentales traslacionales de la estructura en la dirección $X$ y $Y$, respectivamente. Cuando se deba sustituir el valor constante de $Q$ por $Q_{\max }$, en dichos factores se debe sustituir $Q$ por $Q_{\max }(T f)$, por lo que en este caso, para las direcciones $X$ y $Y$ dichos factores se definen como: $F A D_{\max }-X=Q_{\max }(T f x) R\left(T f x, R_{o}\right) \rho$ y $F A D_{\max }-Y=Q_{\max }(T f y) R\left(T f y, R_{o}\right) \rho$, respectivamente.

En el CDS-MDOC-2015 se menciona que se justificará tomar en cuenta los efectos de la interacción suelo-estructura, cuando se cumpla la desigualdad mostrada en la ec. 10

$\frac{T_{e o}}{T_{S}} \frac{H_{S}}{H_{e o}}<2.5$

donde:

$T_{e o}$ es el periodo fundamental de la estructura supuesta con base rígida.

$T_{S}$ es el periodo dominante del terreno equivalente.

$H_{S}$ es el espesor total del estrato de terreno equivalente.

$H_{e o}$ es la altura efectiva fundamental de la estructura supuesta con base rígida. 
Se debe recordar que en el planteamiento para obtener las funciones de $Q_{\max }$ no se consideró la interacción suelo-estructura, por lo que dichas funciones solo aplican cuando de acuerdo a la ec. 10, no se justifique tomar en cuenta los efectos de dicha interacción.

Por otra parte, cabe mencionar que en el CDS-MDOC-2015 no se discute ampliamente como se llegó a establecer el $F_{s e r}=5.5$. Por lo anterior, los autores del presente trabajo consideran que es recomendable realizar un estudio para definir el periodo de retorno óptimo de diseño para el ELS de estructuras tipo edificio ubicadas en el municipio de Veracruz. Con base en este periodo de retorno, puede calcularse el espectro de diseño para el ELS y el correspondiente $F_{\text {ser }}$. Sin embargo, dicho estudio está fuera de los alcances del presente trabajo y constituye otro tema de investigación. Es importante mencionar que aunque se considere un espectro para el ELS obtenido con el periodo de retorno óptimo mencionado, no hay garantía de que no se siga presentando la problemática expuesta.

\section{EJEMPLOS ILUSTRATIVOS}

La estructura a diseñar es tipo edificio de 12 niveles conformada por marcos de concreto reforzado, y de acuerdo con las recomendaciones del CDS-MDOC-2015 cumple con el conjunto de requisitos para diseñarla con $Q=4$. Por la altura de la estructura y su sistema estructural se estima que sus periodos fundamentales en ambas direcciones traslacionales serán mayores que un segundo. La estructura se ubica en un sitio del municipio de Veracruz cuyo terreno se clasificó hipotéticamente como tipo II, de acuerdo a las recomendaciones del CDS-MDOC-2015. Posteriormente se mostrará que en el análisis de esta estructura se justifica no tomar en cuenta los efectos de la interacción suelo-estructura. Considerando lo discutido en relación a la fig. 2, los EDM de esta estructura se intersectarán con el ED-ELS y el $T_{e i}$ será menor que ambos periodos fundamentales traslacionales de la estructura, por lo que es posible que sus Vb-diseño en ambas direcciones sean menores que los correspondientes Vb-ELS. Debido a esto, la estructura se diseñó para dos EDM: uno considerando $Q^{\prime}\left(T_{e}, Q=4\right)$, al cual se le denomina EDM-A y otro considerando $Q^{\prime}\left(T_{e}, Q_{\max }\right)$, al que se le denomina EDM-B. A la estructura diseñada con el EDM-A se le denomina estructura-A y a la diseñada con el EDM-B se le denomina estructura-B. El análisis y diseño sísmico de las estructuras se realizó de acuerdo a las recomendaciones del CDS-MDOC-2015. El diseño estructural y el detallado de los miembros estructurales se realizaron de acuerdo a los lineamientos de las Normas Técnicas Complementarias para Diseño y Construcción de Estructuras de Concreto del Reglamento de Construcciones de la Ciudad de México de 2017 (NTC-DC-2017). Se pretende mostrar, a partir de la respuesta inelástica de ambas estructuras, que cuando actúe el sismo de diseño correspondiente al ELS; solo la estructura-B no desarrollará la suficiente sobrerresistencia o incluso ductilidad como para que su respuesta sea inelástica. A continuación se detallan todos los aspectos necesarios para realizar el diseño sísmico de las estructuras.

\section{Características y propiedades de la estructura}

La estructura es tipo edificio para uso de oficinas conformada por marcos no contraventeados de concreto reforzado, cuya configuración geométrica en planta se muestra en la fig. 4. Tiene cuatro crujías en ambas direcciones. El ancho de sus crujías en la dirección $X$ es de $6.0 \mathrm{~m}$ y en la dirección $Y$ de $5.0 \mathrm{~m}$, por lo que las dimensiones de su base en las direcciones $X$ y $Y$ son $24 \mathrm{~m}$ y $20 \mathrm{~m}$, respectivamente. La estructura tiene 12 niveles, su primer nivel tiene una altura de $4 \mathrm{~m}$ y los restantes de $3 \mathrm{~m}$. Su área total construida es de $5760 \mathrm{~m}^{2}$ y tiene una altura total de $37 \mathrm{~m}$. Con este conjunto de características, la estructura cumple los requisitos para ser clasificada con una importancia estructural B1 de acuerdo a la sección 3.1.2 de las recomendaciones del CDS-MDOC-2015. 


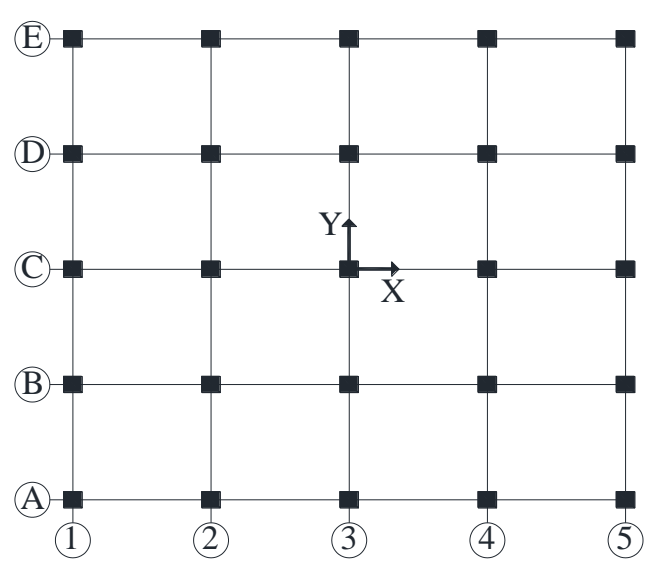

Figura 4. Configuración geométrica en planta de la estructura

Las dimensiones y forma de la estructura en planta son las mismas en todos los niveles, y no tiene entrantes ni salientes. El sistema de piso es a base de losa maciza de concreto reforzado colada monolíticamente con sus vigas de apoyo, y no tiene aberturas en ningún nivel (se ignoran los espacios para ascensor o escaleras). El peralte total de la losa en todos los niveles es el mismo y excede ligeramente el mínimo para no revisar deflexiones ante cargas muertas y vivas. Se establece la hipótesis de que el sistema de piso es lo suficientemente rígido y resistente ante cargas laterales, y por lo tanto se comporta como un diafragma horizontal rígido. Como se puede ver en la fig. 4 los marcos resistentes a carga lateral son simétricos respecto a ambos ejes ortogonales del edificio. Los cambios de las dimensiones de secciones de vigas y columnas en la altura de la estructura se realizaron de manera gradual y simétrica respecto a ambos ejes ortogonales, por lo que la distribución de masas en planta debido al peso propio de los miembros es uniforme y en altura varía gradualmente. La distribución de masas en planta debido a cargas vivas es uniforme en todos los niveles, y la magnitud de las cargas vivas es diferente solo en el nivel superior. Debido a la distribución simétrica tanto de las masas en todas las plantas por cargas muertas y vivas, como de las rigideces en ambas direcciones en todos los entrepisos; la excentricidad torsional es teóricamente nula y en el análisis sísmico solo se considera la excentricidad accidental. Con este conjunto de propiedades y características, la estructura cumple los requisitos para que pueda ser clasificada como regular de acuerdo a la sección 3.3.2.1 de las recomendaciones del CDS-MDOC-2015, y por lo tanto aplica $\alpha=1$.

En cuanto al sistema resistente a fuerzas laterales de la estructura, la resistencia en todos los entrepisos es suministrada únicamente por marcos no contraventeados de concreto reforzado. Existen muros divisorios y de ventanas de fachada pero ambos están desligados de la estructura. Debido a la distribución significativamente uniforme de las masas y de la rigidez lateral en la altura de la estructura, y por ende en las capacidades resistentes de los entrepisos; es posible mostrar que las estructuras diseñadas cumplen con el requisito tres de la sección 3.3.1.1 de las recomendaciones del CDS-MDOC-2015. Además, los miembros de los marcos se diseñaron y detallaron para cumplir con los requisitos del capítulo nueve: diseño de estructuras de ductilidad alta, de las NTC-DC-2017. Con este conjunto de propiedades y consideraciones, la estructura cumple los requisitos especificados en la sección 3.3.1.1 de las recomendaciones del CDSMDOC-2015, para que pueda ser diseñada con $Q=4$ en ambas direcciones.

La estructura tiene cinco marcos en ambas direcciones principales de análisis, y cada marco tiene cuatro crujías, por lo que de acuerdo a la sección 3.3.1.4 de las recomendaciones del CDS-MDOC-2015; cumple los requisitos para considerar un valor de $\rho=1.25$ en ambas direcciones de análisis.

Por tratarse de una estructura de concreto reforzado el valor del amortiguamiento estructural es $\zeta_{e}=5 \%$ y el correspondiente valor del factor de amortiguamiento es $\beta=1$. 


\section{Cálculo del espectro de diseño transparente regional}

La estructura se considera ubicada hipotéticamente en un sitio del municipio de Veracruz. En el planteamiento para obtener las funciones de $Q_{\max }$ se pudo observar que fue posible obtenerlas debido a que los espectros regionales están definidos por una forma funcional. Sin esta característica de los espectros regionales, no hubiera sido posible obtener dichas funciones. En el CDS-MDOC-2015 se menciona que los espectros regionales son conservadores, por lo que sería deseable usar un espectro especifico de sitio, construido de acuerdo con los lineamientos del CDS-MDOC-2015; los cuales tienen la misma forma funcional que los espectros regionales. Sin embargo, por razones económicas, no fue posible construir dicho espectro para implementarlo en el estudio. Por otra parte, los autores del presente estudio consideran que los suelos del municipio de Veracruz no son tan firmes como el terreno tipo I que se especifica en el CDSMDOC-2015, ni tan blandos como el terreno tipo III, por lo que se optó usar el terreno tipo II. De acuerdo con lo anterior, en el programa PRODISIS versión 4.1, el cual forma parte del CDS-MDOC-2015, se introdujeron los siguientes datos: (1) las coordenadas geográficas del sitio referido, (2) la importancia de la estructura a diseñar (B1), (3) para el depósito de suelo en el que se desplanta la estructura, idealizado como un estrato homogéneo equivalente; los siguientes valores hipotéticos de su velocidad de ondas de corte, $\mathrm{Vs}=600 \mathrm{~m} / \mathrm{s}$, y su espesor, Hs=30 m. Como resultado de estas acciones dicho programa ubicó el sitio en la zona sísmica B, clasificó el suelo del sitio como terreno tipo II y calculó el correspondiente espectro de diseño transparente regional mostrado en la fig. 2.

\section{Principales consideraciones adicionales para el análisis y diseño de las estructuras}

Para el análisis y el diseño de la estructura se realizaron las siguientes consideraciones adicionales: (1) las propiedades mecánicas de los materiales empleados cumplen con los lineamientos de las NTC-DC2017. Se utilizó concreto clase 1 con una resistencia especificada a compresión, $f^{\prime} c=350 \mathrm{kgf} / \mathrm{cm}^{2}$, y con un módulo de elasticidad, $E c=14000 \sqrt{f^{\prime} c}$, y acero de refuerzo con esfuerzo de fluencia, $f y=4200 \mathrm{kgf} / \mathrm{cm}^{2}$; (2) el modelado de los miembros estructurales cumple con los lineamientos de las NTC-DC-2017. Las secciones transversales de las vigas interiores y de borde se modelaron como vigas "T" y "L invertida", respectivamente. Para tomar en cuenta la pérdida de rigidez debida al agrietamiento en vigas y columnas, se consideraron las siguientes fracciones del momento de inercia grueso $\left(I_{g}\right)$ de sus secciones trasversales: $0.5 I_{g}$ y $0.7 I_{g}$, respectivamente; (3) la carga muerta de las losas de entrepiso incluye los siguientes pesos propios: de la losa, de una capa de mortero, de un recubrimiento de loseta cerámica, del sistema de plafón y de la sobrecarga en losas coladas en su sitio de acuerdo a las NTC-DC-2017. La carga muerta de la losa de azotea incluye los siguientes pesos propios: de la losa, de dos capas de mortero, de un relleno para dar pendiente a la superficie de la losa, de un sistema impermeabilizante, del sistema de plafón y de la sobrecarga en losas coladas en su sitio de acuerdo a las NTC-DC-2017. Los pesos propios de vigas y columnas los calculó el programa empleado para el análisis estructural y los revisaron los autores del presente trabajo; (4) las intensidades de las cargas vivas: máxima, instantánea y media consideradas, son las especificadas en las Normas Técnicas Complementarias sobre Criterios y Acciones para el Diseño Estructural de las Edificaciones del Reglamento de Construcciones de la Ciudad de México de 2017 (NTC-CA-2017), para edificaciones con uso de oficinas; (5) los diferentes tipos de combinaciones de carga de diseño y los factores de carga que en ellas intervienen, son las especificadas en las NTC-CA-2017; (6) para el análisis sísmico de las estructuras se consideraron la mayoría de los requisitos especificados en las recomendaciones del CDSMDOC-2015. Debido a que la estructura se ubica en la zona sísmica B, no es necesario considerar el efecto de la componente vertical de los sismos de diseño. El análisis sísmico de las estructuras se efectuó con el programa para análisis y diseño de edificios ECOgcW3 (Corona, 2017), y consistió de un análisis dinámico modal espectral, que es uno de los métodos de análisis que se especifica en el CDS-MDOC-2015 para estructuras tipo edificio. En los análisis, los efectos de segundo orden y la combinación de efectos bidireccionales de las componentes horizontales ortogonales de los sismos de diseño, se calcularon conforme a dicho programa. Cabe mencionar que este programa calcula la combinación de los efectos 
bidireccionales ortogonales de acuerdo al criterio $\pm 100 \%$ sismo en dirección $X \pm 30 \%$ sismo en dirección $Y$ y $\pm 100 \%$ sismo en dirección $Y \pm 30 \%$ sismo en dirección $X$, y no mediante la raíz cuadrada de la suma de los cuadrados de sus efectos, como lo indican las recomendaciones del CDS-MDOC-2015. Sin embargo, se piensa que esta discrepancia no afectará lo que se pretende mostrar en el presente trabajo, ya que si ambos cortantes basales, Vb-ELS y Vb-diseño, se calculan con el mismo criterio de combinación de efectos bidireccionales, el cociente entre ambos cortantes basales será el mismo. La excentricidad accidental y la revisión del cortante basal mínimo en el ELPC, se consideraron de acuerdo a las recomendaciones del CDSMDOC-2015. (7) la distorsión de entrepiso permisible considerada para el ELS es de 0.004 (elementos no estructurales separados de la estructura), y para el ELPC de 0.030 (marcos dúctiles de concreto reforzado diseñados con $Q=4$ ). Ambas se tomaron de las recomendaciones del CDS-MDOC-2015; (8) el cálculo de las cuantías requeridas de refuerzo longitudinal y transversal de los miembros se realizó con el apoyo del programa $\mathrm{ECOgcW} 3$, y el detallado de estos refuerzos lo realizaron los autores del presente trabajo.

\section{Diseño de la estructura-A}

El diseño inició con un dimensionamiento preliminar de las secciones transversales de los miembros, propuesto con base en la experiencia práctica de los autores del presente estudio, y que cumple con los requisitos geométricos especificados en el capítulo nueve: diseño de estructuras de ductilidad alta, de las NTC-DC-2017. Con este dimensionamiento y con las cargas muertas y vivas reglamentarias, se elaboró un modelo matemático de la estructura con su base rígida, al cual se le efectuó un análisis modal para conocer sus propiedades dinámicas. Con la información obtenida de este modelo y las propiedades dinámicas del depósito de suelo en el que se desplanta la estructura, idealizado como un estrato homogéneo equivalente; se aplicó la ec. 10 para establecer si se justifica tomar en cuenta los efectos de la interacción suelo-estructura (ISE). En la tabla 1 se muestran las propiedades dinámicas del suelo y del modo fundamental de la estructura, estas últimas proporcionadas por el programa usado para los análisis símicos; así como el resultado de la aplicación de la ec. 10 a partir de estos datos. Se observa que en este primer análisis no se justifica tomar en cuenta los efectos de la ISE.

A continuación se inició el dimensionamiento por rigidez lateral de la estructura, considerando el EDELS y el EDM-A mostrados en la fig. 5. Ambos espectros se calcularon a partir del espectro transparente regional obtenido anteriormente. El EDM-A se calculó para los siguientes valores de los parámetros de diseño sísmico aplicables en ambas direcciones traslacionales: $\alpha=1, \rho=1.25$ y $R_{o}=2.5$ que es el valor correspondiente de este parámetro para marcos de concreto reforzado que se diseñan para $Q=4$. En la fig. 5 se observa que el $T_{e i}=0.35 \mathrm{~s}$, y que después de este periodo las ordenadas del EDM-A son menores que las ordenadas del ED-ELS.

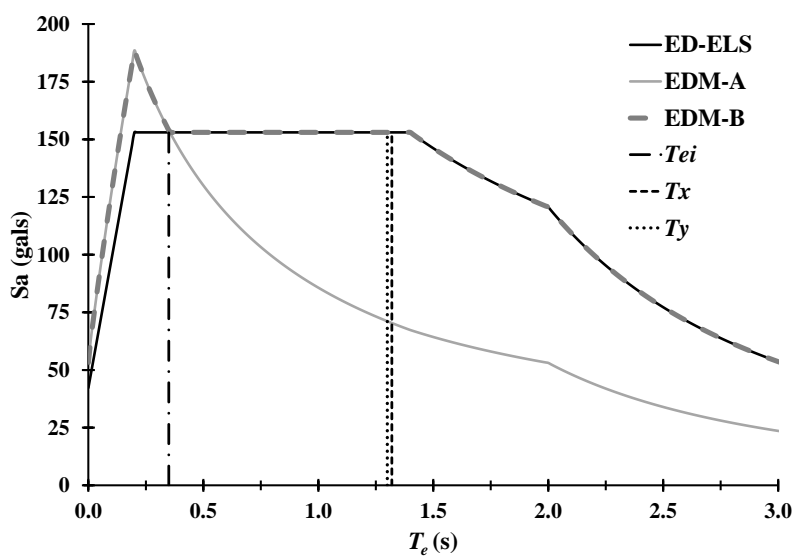

Figura 5. Espectros utilizados para el diseño sísmico de las estructuras. 
El dimensionamiento por rigidez lateral se llevó a cabo mediante el siguiente procedimiento iterativo: se incrementaron las secciones transversales de los miembros propuestas en el dimensionamiento preliminar y se le efectuaron simultáneamente a la estructura dos análisis sísmicos; uno con el ED-ELS y otro con el EDM-A. Con los resultados del análisis modal se aplicó la ec. 10 para establecer nuevamente si se justifica tomar en cuenta los efectos de la ISE. Se verificó que no se justificaba tomar en cuenta dichos efectos y se continuó con el procedimiento. A partir de los desplazamientos laterales relativos de pisos consecutivos obtenidos directamente del análisis con el ED-ELS, se calcularon las máximas distorsiones en ambas direcciones y estas se compararon con la distorsión permisible para el ELS. De forma similar, a partir de los desplazamientos laterales relativos de pisos consecutivos obtenidos del análisis con el EDM-A, multiplicados por $F A D-X$ o $F A D-Y$ según la dirección de análisis; se calcularon las máximas distorsiones en ambas direcciones, y estas se compararon con la distorsión permisible para el ELPC. Si las máximas distorsiones en algún estado límite eran mayores que la permisible para dicho estado límite, se incrementaban las secciones de los miembros, y si eran menores que la permisible se disminuían las secciones. En ambos casos se actualizaban las masas reactivas en los sistemas de piso y la rigidez lateral de la estructura, se aplicaba la ec. 10 para establecer nuevamente si se justificaba tomar en cuenta los efectos de la ISE, se observaba que no era justificable; se efectuaban nuevamente los dos análisis sísmicos de manera simultánea para calcular las máximas distorsiones y compararlas con la permisible para el respectivo estado límite. Esta serie de análisis y procesamiento de sus resultados se llevaron a cabo hasta que las máximas distorsiones en ambas direcciones para alguno de los dos estado límite, fueron aproximadamente iguales a la permisible para dicho estado límite. En la tabla 1 se muestran las propiedades dinámicas del modo fundamental de la estructura para el último análisis, así como el resultado de la aplicación de la ec. 10 a partir de estos datos. Se observa que en este último análisis también no se justifica tomar en cuenta los efectos de la ISE.

Tabla 1. Aplicación de la ec. 10 para establecer la justificación de considerar los efectos de la ISE en las estructuras A y B

\begin{tabular}{|c|c|c|c|c|c|c|c|}
\hline \multicolumn{3}{|c|}{ Pro } & edades dinámicas & & & & \multirow{3}{*}{$\begin{array}{l}\text { Resultado } \\
\text { de la ec. } 10\end{array}$} \\
\hline \multicolumn{3}{|c|}{ Del estrato equivalente de suelo } & \multicolumn{4}{|c|}{ De los modos fundamentales de la estructura } & \\
\hline $\begin{array}{l}H_{s} \\
(\mathrm{~m})\end{array}$ & $\begin{array}{c}V_{s} \\
(\mathrm{~m} / \mathrm{s})\end{array}$ & $\begin{array}{l}T_{s} \\
(\mathrm{~s})\end{array}$ & & & $\begin{array}{l}H_{e o} \\
(\mathrm{~m})\end{array}$ & $\begin{array}{l}T_{e o} \\
(\mathrm{~s})\end{array}$ & \\
\hline \multirow{4}{*}{30} & \multirow{4}{*}{600} & \multirow{4}{*}{0.2} & \multirow{2}{*}{ Análisis modal inicial } & "X" & 24.20 & 1.78 & $11.03>2.5$ \\
\hline & & & & "Y" & 23.75 & 1.74 & $10.99>2.5$ \\
\hline & & & \multirow{2}{*}{ Análisis modal final } & "X" & 25.07 & 1.32 & $7.898>2.5$ \\
\hline & & & & "Y" & 24.98 & 1.30 & $7.806>2.5$ \\
\hline
\end{tabular}

Es importante enfatizar que, como se mencionó, en cada uno de los análisis sísmicos requeridos durante el proceso iterativo para el dimensionamiento por rigidez lateral de la estructura, se aplicó la ec. 10 para establecer si se justificaba tomar en cuenta los efectos de la ISE, y en ninguno de los análisis se justificó. En dicho proceso iterativo se requirieron varios análisis, pero por razones de brevedad, en la tabla 1 solo se muestran los datos y resultados de los análisis inicial y final.

En la fig. 6 se muestran las envolventes de las distorsiones de entrepiso resultantes del dimensionamiento por rigidez lateral de la estructura. Dichas envolventes corresponden al eje de columnas definido por la intersección de los ejes estructurales 5 y E mostrados en la fig. 4. Se eligió este eje de columnas porque es uno de los cuatro ejes (los cuatro de las esquinas de la estructura) en donde se presentan las máximas distorsiones en ambas direcciones. En las figs. $6 a$ y $6 \mathrm{~b}$ se puede observar que el ELS rigió la rigidez lateral de la estructura, ya que las máximas distorsiones en el ELS son más cercanas a la permisible para dicho estado límite $(\approx 90 \%)$, de lo que son las máximas distorsiones en el ELPC a la permisible para este estado límite $(\approx 73 \%)$. 


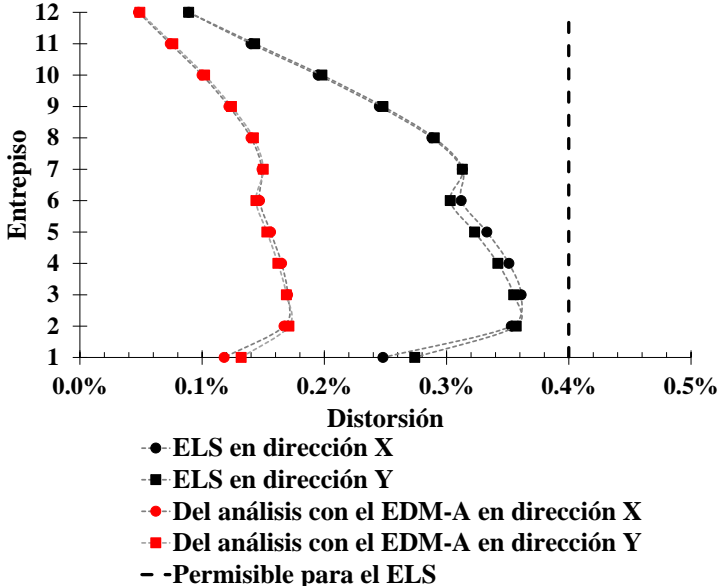

a) En el ELS y obtenidas del análisis con el EDM-A

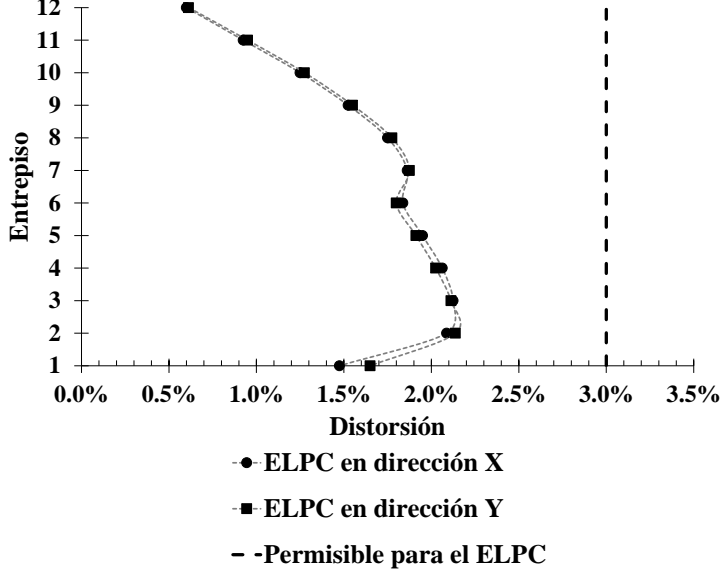

b) Para el ELPC

Figura 6. Envolventes de distorsiones de entrepiso en la estructura-A

Los periodos fundamentales traslacionales de la estructura resultantes del dimensionamiento por rigidez lateral son: $T f x=1.32 \mathrm{~s}$ y $T f y=1.30 \mathrm{~s}$. Ambos periodos son mayores que el $T_{e i}$ y $T_{a}$. Debido a lo anterior, el valor de $R$ es igual a $R_{o}$ en ambas direcciones, por lo que los valores de los FAD son: $F A D-X=$ $F A D-Y=(Q=4)\left(R_{o}=2.5\right)(\rho=1.25)=12.5$. El valor del producto $\rho R$ el cual como se mencionó, puede interpretarse como la sobrerresistencia total, obtenido en ambas direcciones de análisis es de 3.125. En la tabla 2 se muestran las dimensiones globales requeridas de las secciones transversales de las vigas y columnas (dimensión menor y mayor para columnas, base y peralte para vigas).

Tabla 2. Dimensiones y cuantías de refuerzo colocadas para las secciones transversales de los miembros de las estructuras

\begin{tabular}{ccccccc}
\hline & Nivel & $\begin{array}{c}\text { Dimensiones globales } \\
\text { de las secciones }(\mathrm{cm}) \\
\text { Estructuras A y B }\end{array}$ & \multicolumn{2}{c}{ Cuantías de refuerzo longitudinal (\%) } \\
& $1-6$ & $70 \times 80$ & Estructura-A & Estructura-B \\
\hline \multirow{2}{*}{ Columnas } & $1-12$ & $60 \times 70$ & 1.09 & De 1.43 a 2.76 \\
& $7-6$ & \multicolumn{2}{c}{1.21} & \multicolumn{2}{c}{1.21} \\
\hline \multirow{2}{*}{ Vigas } & $1-6$ & $35 \times 60$ & Lecho superior & Lecho inferior & Lecho superior & Lecho inferior \\
\cline { 3 - 7 } & $7-12$ & $30 \times 60$ & De 0.97 a 1.09 & De 0.53 a 0.67 & De 1.88 a 2.14 & De 1.19 a 1.61 \\
& & & De 0.85 a 0.96 & De 0.42 a 0.74 & De 0.96 a 1.74 & De 0.61 a 1.09 \\
\hline
\end{tabular}

En la fig. 6a también se muestran las envolventes de las distorsiones de entrepiso obtenidas del análisis con el EMD-A, las cuales son menores que las correspondientes en el ELS. En la tabla 3 se muestran los cortantes basales en ambas direcciones para ambos estados límite, donde se observa que los cortantes basales en el ELS son mayores que los cortantes basales de diseño. Los dos resultados anteriores muestran que el ED-ELS impone a la estructura demandas de fuerza y de deformación lateral mayores que el EDMA, como era de esperarse. En la tabla 3 también se muestran los valores del cociente $\mathrm{Vb}$-ELS/Vb-diseño para ambas direcciones. Estos resultados indican que para que la estructura alcance el Vb-ELS en ambas direcciones, debe desarrollar una fracción significativa de su sobrerresistencia total $[(\approx 2 / 3.125) * 100=64 \%]$, y su respuesta puede ser inelástica.

Tabla 3. Cortantes basales en ambos estados límite de la estructura-A

\begin{tabular}{lccc}
\hline & Vb-ELS & Vb-diseño & Cociente Vb-ELS/Vb-diseño \\
\hline Dirección X & 848.53 & 403.22 & 2.10 \\
Dirección Y & 854.77 & 410.56 & 2.08 \\
\hline
\end{tabular}


Una vez realizado el dimensionamiento por rigidez lateral de la estructura se realizó su diseño por resistencia, que consiste en proporcionales a los miembros tanto las áreas de refuerzo longitudinal y transversal para resistir las fuerzas internas obtenidas del análisis con el EDM-A, como el detallado de ambos tipos de refuerzo. Para esto paralelamente se verificó que las dimensiones de las secciones fueran adecuadas para resistir la fuerza cortante de diseño y para albergar las cantidades de refuerzo requeridas cumpliendo las cuantías permisibles y las separaciones horizontales libres mínimas. En la fig. 7 se muestran los armados de las secciones extremas tipo de vigas y columnas resultantes del diseño, y en la tabla 2 se muestra un resumen de las cuantías de refuerzo longitudinal colocadas.

columnas de planta baja

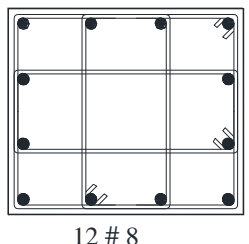

3E\#4: @9 corrido

trabes nivel 2

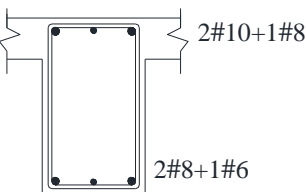

1E\#3: 1@5,14@9, resto @14 c/e

trabes nivel 6
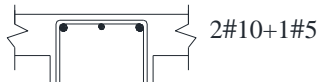

- $2 \# 8$

E\#3:1@5,10@12,resto @14c/e columnas niveles 2 a 6

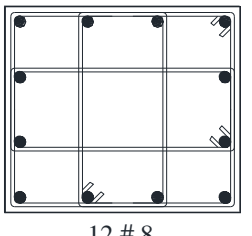

3E\#4: 9@9, resto@14 c/e

trabes nivel 3 columnas niveles 7 a 12

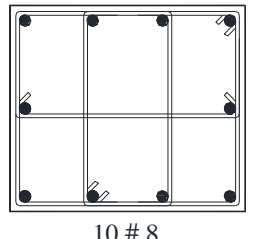

2E\#4+1G\#4: 10@8,resto@15 c/e

trabes nivel 4

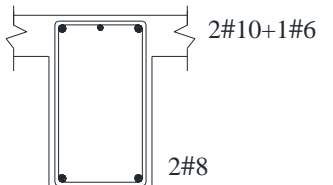

1E\#3: 1@5,12@10, resto @ 14 c/e

trabes nivel 8

trabes nivel 7

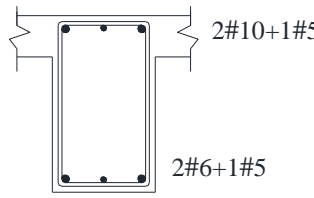

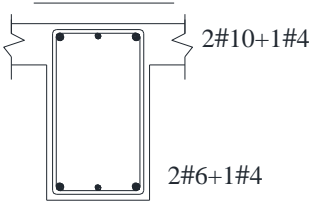

1E\#3: 1@5,12@10, resto@14 c/e trabes nivel 1

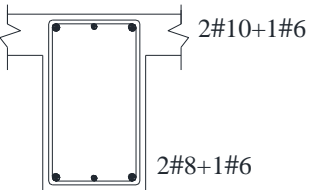

1E\#3: 1@5,15@8, resto@14 c/e

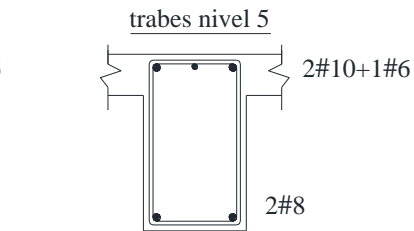

1E\#3: 1@5, 12@10, resto @14 c/e

trabes niveles 9 a 12

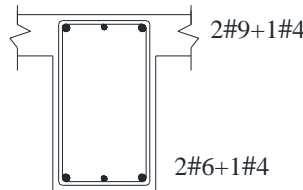

Figura 7. Armado de las secciones transversales extremas tipo de vigas y columnas de la estructura-A

\section{Diseño de la estructura-B}

Esta estructura se diseñó de inicio a fin de la misma forma que la estructura-A, y debido a que tienen la misma geometría, también se determinó que no se justificaba tomar en cuenta los efectos de la ISE a lo largo del proceso iterativo del diseño. Por lo anterior, se consideró el EDM-B mostrado en la fig. 5. Este espectro se calculó para los siguientes valores de los parámetros de diseño sísmico aplicables en ambas direcciones traslacionales: $\alpha=1, \rho=1.25, Q=4$ o $Q=Q_{\max }$ según que $T_{e}$ sea menor o igual, o mayor que $T_{e i}$, respectivamente; y $R_{o}=2.5$. Cabe mencionar que se consideró el valor de $R_{o}$ correspondiente a marcos de concreto reforzado diseñados para $Q=4$ debido a que, como se mencionó, los miembros estructurales diseñados cumplen los requisitos de detallado para adoptar dicho valor de $Q$, independientemente del valor de $Q_{\max }$ que se obtenga. Ambos espectros se muestran en la fig. 5. En esta figura se observa que hasta el $T_{e i}$ las ordenadas del EDM-A y del EDM-B son iguales y que después del $T_{e i}$ las ordenadas del EDM-B y del ED-ELS son iguales.

En la fig. 8 se muestra las gráficas del $F_{\text {ser }}$ y de los $F R T$ del EDM-A y del EDM-B. Se puede observar que para periodos menores e igual al $T_{e i}=0.35 \mathrm{~s}$ los $F R T$ de ambos EDM son menores que el $F_{s e r}$, y que para periodos mayores que el $T_{e i}$ el $F R T$ del EDM-A y del EDM-B es, respectivamente, mayor e igual que el $F_{\text {ser. }}$ 


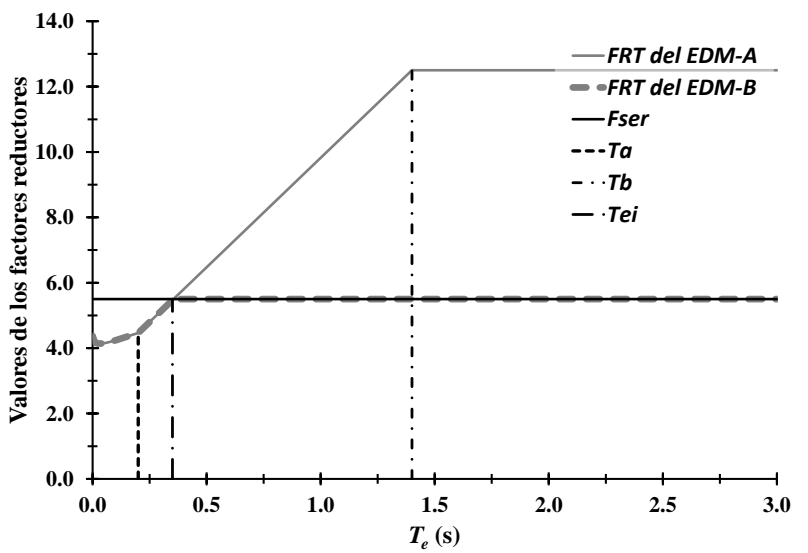

Figura 8. Factores reductores de los espectros de diseño de las estructuras diseñadas

En la fig. 9 se muestra la gráfica de $Q_{\max }$ en función del periodo estructural. Se observa que, como se mencionó, para periodos menores e igual al $T_{e i}=0.35 \mathrm{~s}$ se debe tomar el valor de diseño de $Q$ aplicable a la estructura, $Q=4$. Para periodos mayores que el $T_{e i}$ el valor de $Q_{\max }$ decrece, como se requiere, y a partir de $T_{b}=1.4$ el valor de $Q_{\max }$ permanece constante e igual a 1.76 .

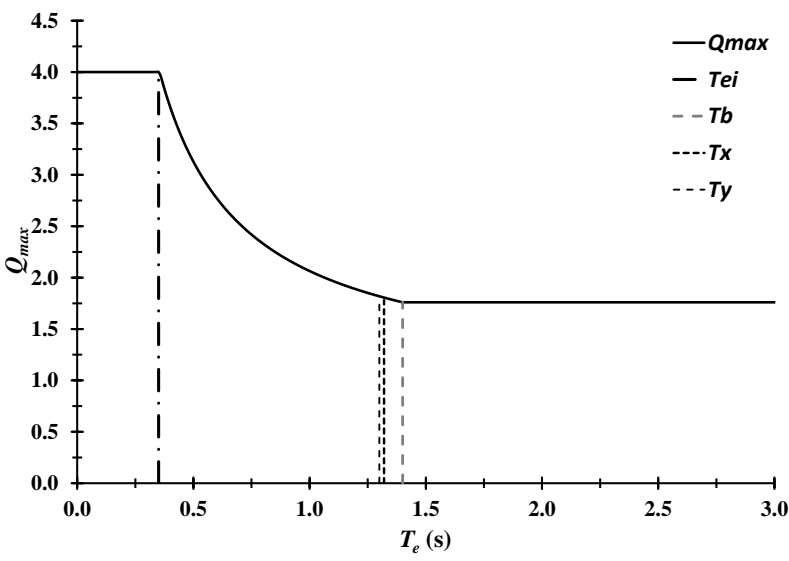

Figura 9. Gráfica de $Q_{\max }$ en función del periodo estructural

Al realizar el dimensionamiento por rigidez lateral de la estructura-B se requirieron las mismas dimensiones de las secciones transversales de vigas y columnas de la estructura-A, y por lo tanto ambas estructuras tienen los mismos periodos. Este resultado indica que también para la estructura-B el dimensionamiento por rigidez lateral fue regido por el ELS. En la fig. 10 se muestran las envolventes de las distorsiones de entrepiso resultantes del dimensionamiento por rigidez lateral de la estructura-B. Dichas envolventes corresponden al eje de columnas definido por la intersección de los ejes estructurales 5 y E mostrados en la fig. 4. Las distorsiones de entrepiso para el ELPC mostradas en la fig. 10b, se obtuvieron a partir de los desplazamientos laterales relativos de pisos consecutivos obtenidos del análisis con el EDM$\mathrm{B}$, multiplicados por $F A D_{\max }-X$ o $F A D_{\max }-Y$ según la dirección de análisis. Los valores resultantes de dichos factores son: $\quad F A D_{\max }-X \quad=\left(Q_{\max }=1.806\right)\left(R_{0}=2.5\right)(\rho=1.25)=5.64 \quad$ y $\quad F A D_{\max }-Y \quad=\left(Q_{\max }\right.$ $1.818)\left(R_{o}=2.5\right)(\rho=1.25)=5.68$. El valor del producto $\rho R$ en ambas direcciones de análisis es de 3.125. Los valores de $Q_{\max }$ en ambas direcciones son menores que $Q=4$, como se esperaba. Considerando este resultado y debido a que ambas estructuras se diseñaron para una capacidad dúctil de deformación lateral de $Q=4$, la estructura-B tendrá, a diferencia de la estructura-A, una reserva de capacidad de deformación lateral. 


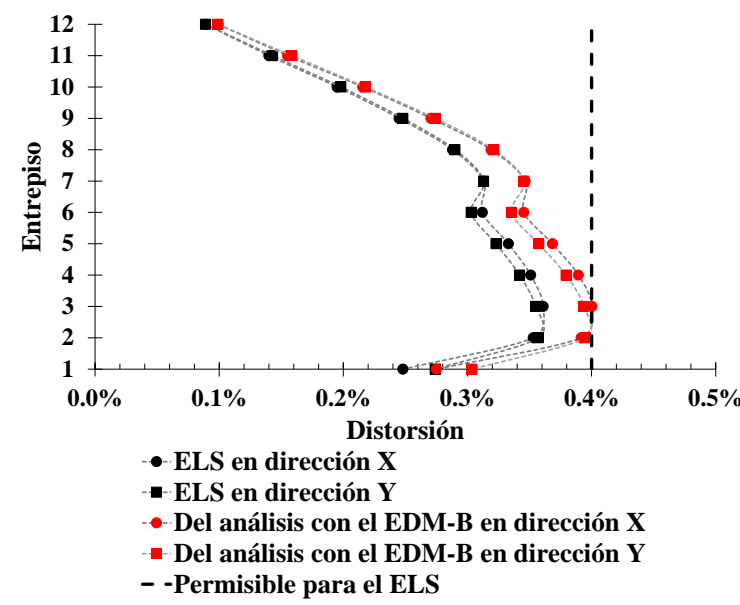

a) En el ELS y obtenidas del análisis con el EDM-B

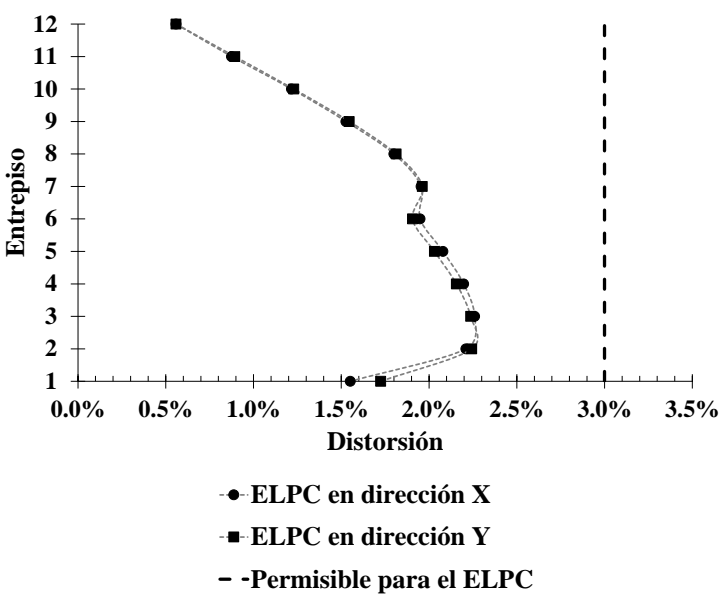

b) Para el ELPC

Figura 10. Envolventes de distorsiones de entrepiso en la estructura-B

En la fig. 10a también se muestran las envolventes de las distorsiones de entrepiso obtenidas del análisis con el EMD-B, las cuales son ligeramente mayores que las correspondientes en el ELS. En la tabla 4 se muestran los cortantes basales en ambas direcciones para ambos estado límite, donde se observa que los cortantes basales de diseño son ligeramente mayores que los correspondientes en el ELS. Los dos resultados anteriores muestran que el EDM-B impone a la estructura demandas de fuerza y de deformación lateral mayores que las impuestas por el ED-ELS. De hecho, la relación de las demandas obtenidas del EDM-B a las demandas obtenidas del ED-ELS son aproximadamente iguales a 1.1, que es el factor de carga aplicable a la combinación de carga de diseño para el ELPC en la que intervienen cargas muertas, vivas y accidentales, de acuerdo a las NTC-CA-2017. En la tabla 4 también se muestran los valores del cociente Vb-ELS/Vb-diseño para ambas direcciones. Estos valores son aproximadamente el inverso del factor de carga 1.1, y estos resultados indican que los Vb-ELS en ambas direcciones son ligeramente menores que los respectivos Vb-diseño, que es el escenario deseable.

Tabla 4. Cortantes basales en ambos estados límite de la estructura-B

\begin{tabular}{lccc}
\hline & Vb-ELS & Vb-diseño & Cociente Vb-ELS/Vb-diseño \\
\hline Dirección X & 848.53 & 933.38 & 0.905 \\
Dirección Y & 854.77 & 940.25 & 0.907 \\
\hline
\end{tabular}

En la fig. 11 se muestran los armados de las secciones tipo de vigas y columnas resultantes del diseño de la estructura-B, y en la tabla 2 se muestra un resumen de las cuantías de refuerzo longitudinal colocadas. Cabe mencionar que también se verificó que las dimensiones de las secciones fueran adecuadas para resistir la fuerza cortante de diseño y para albergar las cantidades de refuerzo requeridas, cumpliendo las cuantías permisibles y las separaciones horizontales libres mínimas. 

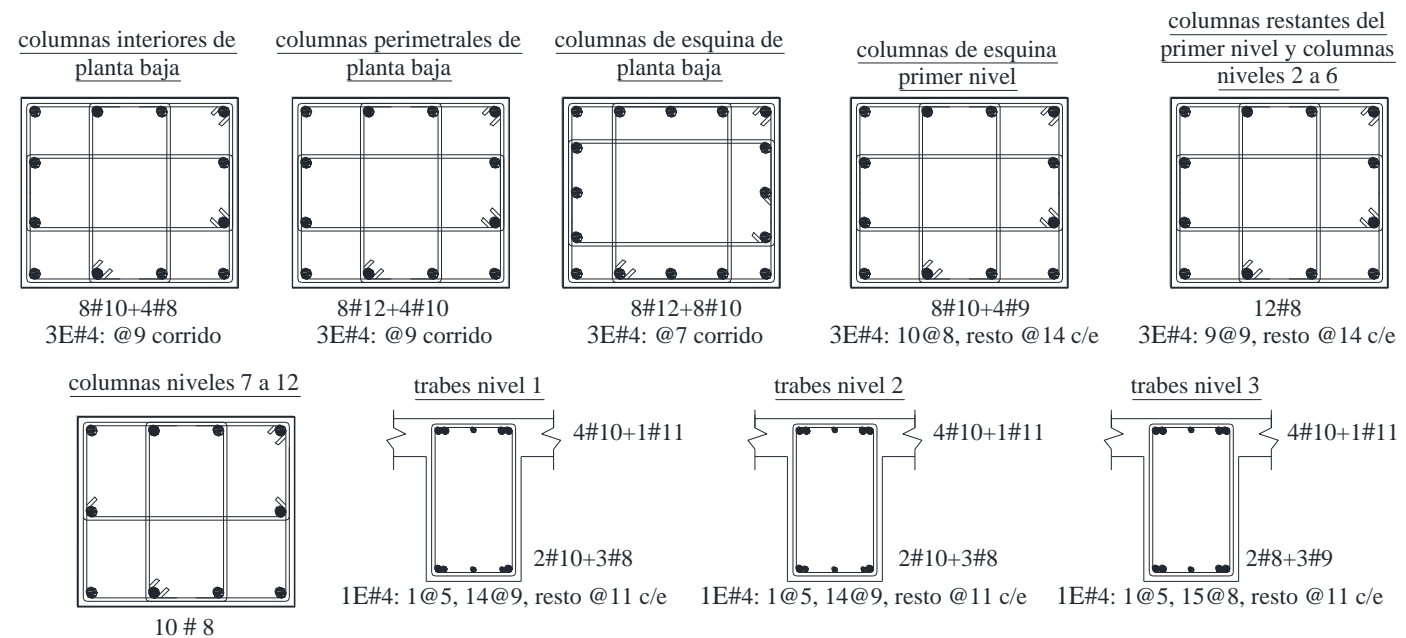

2E\#4+1G\#4: 10@8, resto@ $15 \mathrm{c} / \mathrm{e}$
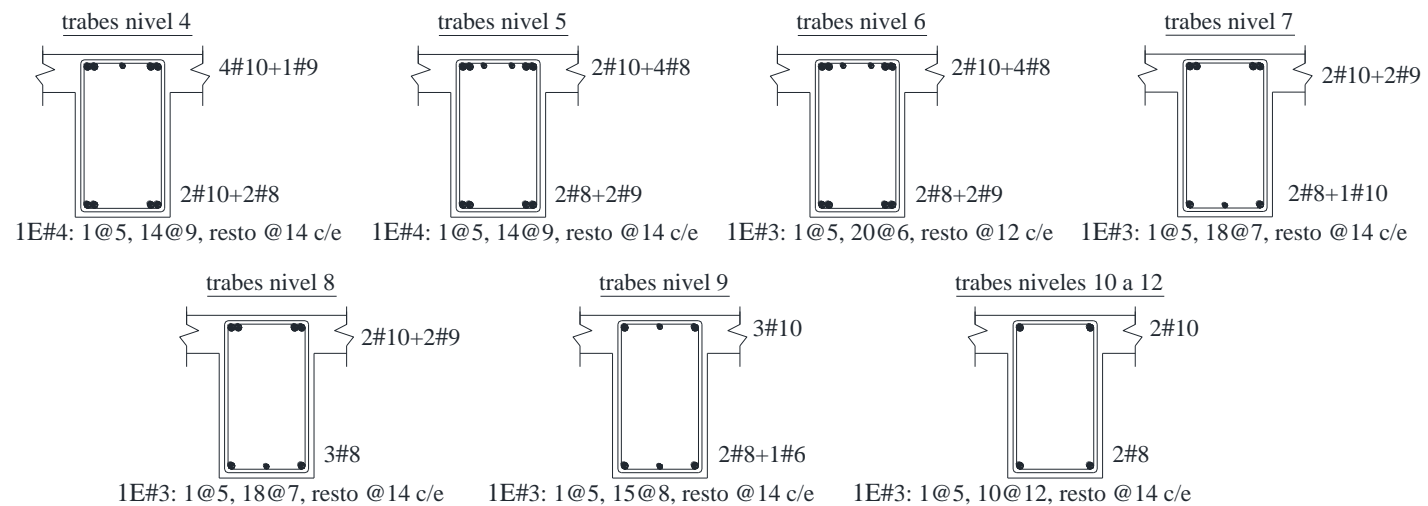

Figura 11. Armado de las secciones transversales extremas tipo de vigas y columnas de la estructura-B

En las estructuras A y B el dimensionamiento por rigidez lateral fue regido por el ELS. En el presente trabajo se define el espectro del indicador de rigidez lateral elástica requerida (IRER) para cada estado límite de ambas estructuras y mediante su comparación se pretende explicar este resultado. Las ordenadas de estos espectros representan el valor del indicador mencionado, y para el ELS se obtienen dividiendo cada una de las ordenadas del ED-ELS entre la distorsión permisible de entrepiso para dicho estado límite. De forma similar, las ordenadas del espectro del IRER para el ELPC se obtienen dividiendo cada una de las ordenadas del EDM, entre el cociente dado por la distorsión permisible de entrepiso para el ELPC entre el producto $Q$ $R\left(T_{e}, R_{o}\right) \rho$. Dicho cociente se puede interpretar como la distorsión de entrepiso permisible asociada al límite del comportamiento elástico de un oscilador de un grado de libertad cuyo periodo de vibrar es $T_{e}$. El nombre de IRER se debe a que el cociente seudoaceleración entre distorsión es análogo a una rigidez lateral, es decir fuerza entre deformación lateral, y a que cuando se desarrollan las distorsiones permisibles de los IRER de ambos estados límite; se consideró que el comportamiento de la estructura debería ser elástico. Es importante mencionar que el IRER propuesto mostrará mejor cuál de los dos estados límite rige el dimensionamiento por rigidez lateral, cuanto mayor sea la contribución del modo fundamental de una estructura a su respuesta total. Pueden existir otros aspectos que influyan pero no se han identificado. Para obtener el espectro del IRER a partir del EDM-A, en el producto $Q R\left(T_{e}, R_{o}\right) \rho$ se consideró el valor constante $Q=4$. De forma similar, para obtener dicho espectro a partir del EDM-B, en el producto mencionado se consideraron los distintos valores que toma la función $Q_{\max }$ en el intervalo completo de periodos, los cuales se muestran en la fig. 9. En la fig. 12 se muestran los tres espectros obtenidos. 


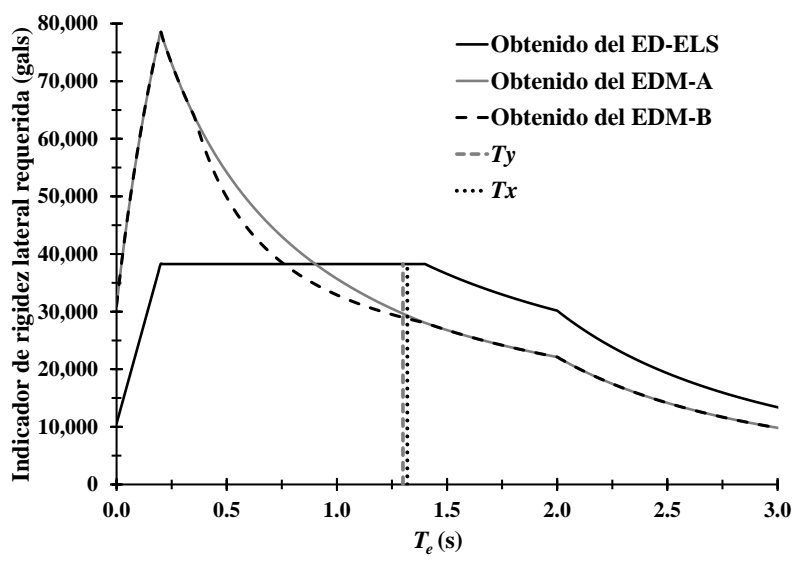

Figura 12. Espectros del indicador de rigidez lateral elástica requerida para los estados límite de diseño

En la fig. 12 las líneas rectas verticales punteadas indican los periodos fundamentales traslacionales de ambas estructuras en las direcciones $X$ y $Y$, y las ordenadas de sus puntos de intersección con los espectros definen los valores del IRER en los respectivos estados límite. Para una estructura con cierto periodo fundamental el estado límite que rige su dimensionamiento por rigidez lateral es el que tiene el mayor valor del IRER. En la fig. 12 se puede observar que para ambos periodos fundamentales traslacionales de las estructuras, el valor del IRER en el ELS es mayor que el correspondiente valor en el ELPC. Este resultado indica que las estructuras requieren mayor rigidez lateral elástica para cumplir con la distorsión permisible correspondiente al ELS, de la que requieren para cumplir con la distorsión permisible correspondiente al ELPC.

\section{Principales consideraciones para el análisis inelástico de las estructuras}

Los modelos matemáticos de ambas estructuras se realizaron en el programa de análisis estructural SAP 2000 v19.2 (CSI, 2017), haciendo las siguientes consideraciones:

Se consideraron las mismas cargas muertas y vivas reglamentarias consideradas en los modelos para los análisis elásticos, así como las mismas combinaciones de carga y los factores de carga que en ellas intervienen.

La rigidez efectiva a flexión y la resistencia de vigas y columnas se obtuvieron de diagramas momento-curvatura $(m-\varphi)$ de sus secciones transversales extremas, idealizados como bilineales, y para el cálculo de dichos diagramas se hicieron las siguientes consideraciones: (1) la geometría, dimensiones y cantidades y distribución del acero de refuerzo longitudinal y transversal consideradas para las secciones, fueron las obtenidas en el diseño; (2) las relaciones esfuerzo-deformación unitaria para el concreto confinado (concreto del núcleo de las secciones) y no confinado (concreto del recubrimiento) se definieron mediante el modelo propuesto por Mander et al., (1988). En dichas relaciones se usó el valor de $f^{\prime} c$ como resistencia del concreto no confinado, en lugar de la resistencia media a compresión del concreto, debido a la falta de resultados de laboratorio para obtener ésta última; (3) la relación esfuerzo-deformación del acero de refuerzo se definió mediante el modelo propuesto por Rodríguez y Botero, (1996). Los valores de los esfuerzos y deformaciones unitarias del acero que definen los diferentes puntos característicos de su curva esfuerzo-deformación, se tomaron como los valores medios esperados de dichas propiedades sugeridos por Rodríguez y Botero, (1996); (4) la carga axial considerada para el cálculo de los diagramas momentocurvatura de las columnas, fue su carga axial de diseño; (5) el momento de fluencia de una sección $\left(M_{y}\right)$ y su correspondiente curvatura $\left(\varphi_{y}\right)$ se definieron como el momento y la curvatura correspondientes a cuando la primera barra de refuerzo longitudinal a tensión alcanza su esfuerzo de fluencia esperado; (6) la curvatura 
máxima o última de una sección $\left(\varphi_{u}\right)$ se definió como la curvatura de menor valor obtenida de las tres condiciones siguientes: (a) el concreto ubicado en la fibra exterior del núcleo de la sección alcanza su deformación unitaria de aplastamiento $\left(\varepsilon_{c u}=0.004\right)$, (b) una de las barras del refuerzo longitudinal a tensión alcanza su deformación unitaria de fractura, (c) la correspondiente a un momento del $85 \%$ del momento máximo obtenido en el diagrama $m-\varphi ;(7)$ el momento último de la sección $\left(M_{u}\right)$ es el correspondiente a $\varphi_{u}$; (8) la primera rama del diagrama momento-curvatura de una sección se define por medio de una línea recta que conecta el origen con el punto definido por $M_{y}$ y $\varphi_{y}$. La segunda rama del diagrama idealizado se define por medio de una línea recta que conecta el punto anterior con el punto definido por $M_{u}$ y $\varphi_{u}$; (9) la pendiente de la primera rama del diagrama define la rigidez elástica efectiva a flexión de la sección transversal ( $\left.E c I_{e f f}\right)$, donde $I_{e f f}$ es el momento de inercia elástico efectivo de la sección transversal, en el cual se considera la contribución del acero de refuerzo a dicha propiedad de la sección. Para cada sección transversal extrema de una viga se calcularon dos diagramas momento-curvatura, uno a momento flexionante negativo y otro a momento flexionante positivo. El $I_{\text {eff }}$ representativo de una sección extrema de una viga se obtiene como el promedio de los $I_{\text {eff }}$ obtenidos de los diagramas a momento negativo y positivo, (10) El $I_{\text {eff }}$ representativo de una viga se obtiene de manera simplista, como el $I_{\text {eff }}$ promedio $\left(I_{\text {eff-prom }}\right)$ de los $I_{\text {eff }}$ representativos de sus secciones extremas. En la tabla 5 se muestran los $I_{\text {eff-prom }}$ de las vigas, normalizados por el momento de inercia de la sección transversal bruta de concreto $I_{g}$; (11) debido a que ambas secciones extremas de las columnas se diseñan, en principio, para el valor absoluto del máximo momento actuante en cada dirección de análisis, el $I_{\text {eff }}$ representativo de una columna se define, de manera simplista, como el $I_{\text {eff }}$ de una de sus secciones transversales extremas. En la tabla 5 se muestran los valores de estos parámetros.

Tabla 5. Relaciones $I_{e f f} / I_{g}$ de las secciones transversales de vigas y columnas de las estructuras

\begin{tabular}{lccc}
\hline & Nivel & Estructura-A & Estructura-B \\
\hline \multirow{2}{*}{ Columnas $\left(I_{\text {eff }} / I_{g}\right)$} & $1-6$ & De 0.24 a 0.43 & De 0.26 a 0.44 \\
& $7-12$ & De 0.17 a 0.34 & De 0.19 a 0.35 \\
\hline \multirow{2}{*}{ Vigas $\left(I_{\text {eff-prom }} / I_{g}\right)$} & $1-6$ & De 0.45 a 0.52 & De 0.76 a 0.96 \\
& $7-12$ & De 0.34 a 0.42 & De 0.35 a 0.70 \\
\hline
\end{tabular}

La inelasticidad en los miembros se consideró concentrada en sus secciones extremas a paño del nudo.

El análisis inelástico realizado a las estructuras consistió en un análisis ante una configuración de cargas laterales monótonas crecientes aplicadas estáticamente, comúnmente denominado análisis "pushover". Para lo que se pretende mostrar en el presente trabajo es necesario determinar el comportamiento de ambas estructuras asociado a su fluencia incipiente y en etapas ligeramente posteriores de su respuesta inelástica. Debido a esto se consideró adecuado utilizar el tipo de análisis inelástico mencionado. Las cargas (desplazamientos) laterales aplicadas en el análisis pushover fueron las correspondientes al modo fundamental de las estructuras en cada dirección. En dicho análisis se consideraron los efectos de segundo orden, las mismas excentricidades de los centros de masas en cada uno de los niveles, y al igual que en el diseño; no se consideró la interacción suelo-estructura.

\section{Resultados del análisis inelástico de las estructuras}

\section{$\underline{\text { Estructura-A }}$}

En la tabla 5 se puede observar que las relaciones $I_{\text {eff-prom }} / I_{g}$ de las vigas estimadas a partir de sus diagramas momento-curvatura, en los cuales se considera explícitamente la contribución del refuerzo longitudinal al $I_{e f f}$, son similares a la especificada por las NTC-DC-2017 para vigas, $I_{e f f} I_{g}=0.5$, mientras que las relaciones $I_{\text {eff }} / I_{g}$ de columnas obtenidas de los diagramas son considerablemente menores que la especificada por dichas normas para columnas, $I_{\text {eff }} I_{g}=0.7$. Debido a esto, la rigidez lateral elástica de la 
estructura en el análisis pushover debe decrecer y sus periodos deben incrementarse. Los periodos fundamentales elásticos de la estructura-A en ambas direcciones traslacionales son: $T f x=1.702 \mathrm{~s}$ y $T f y=1.623$ s. Estos periodos son mayores en un $29 \%$ y $25 \%$, respectivamente, a los correspondientes periodos obtenidos del diseño estructural, $T f x=1.32 \mathrm{~s}$ y $T f y=1.30 \mathrm{~s}$, lo cual era de esperarse.

Del análisis pushover realizado a la estructura para cada dirección de análisis se obtuvieron las correspondientes curvas de respuesta cortante basal-desplazamiento lateral de un punto en la azotea, comúnmente denominada "curva de capacidad". El punto de la azotea monitoreado durante la respuesta es el punto superior del eje de columnas cuyas envolventes de distorsiones se mostraron en los resultados del diseño. En las figs. 13a y 13b se muestran las curvas de capacidad en ambas direcciones. En estas figuras también se muestran dos puntos cuyas ordenadas y abscisas son, respectivamente, cortante basal y el correspondiente desplazamiento lateral del punto de la azotea mencionado, en la correspondiente dirección de análisis; obtenidos de los análisis sísmicos elásticos de la estructura-A considerando el espectro de diseño correspondiente a cada estado límite.

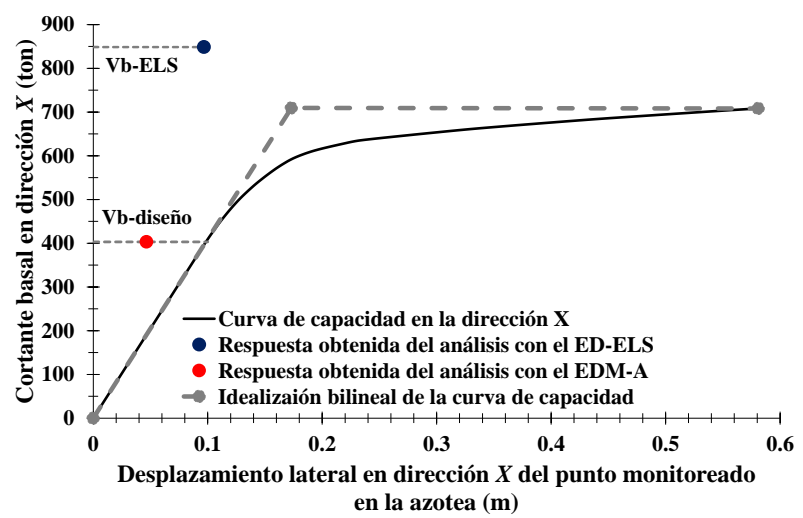

a) En la dirección $X$

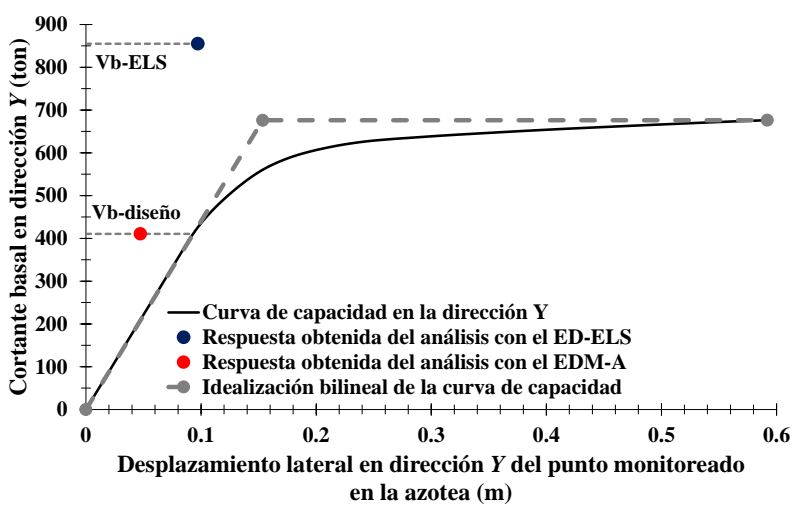

b) En la dirección $Y$

Figura 13. Curvas de capacidad en ambas direcciones de análisis de la estructura-A

En las figs. 13a y 13b se observa que ambos puntos definidos por los parámetros de la respuesta estructural previamente mencionados, están fuera de la curva de capacidad. La primera razón de este resultado es que el método de análisis empleado en el presente trabajo para el diseño de la estructura, especificado por el CDS-MDOC-2015 para el análisis sísmico de estructuras tipo edificio; considera que el comportamiento de una estructura es completamente elástico independientemente de la magnitud de las demandas de resistencias lateral impuestas por un sismo. La segunda razón es que la rigidez lateral elástica de la estructura obtenida considerando la contribución del refuerzo longitudinal en los miembros, fue menor que la rigidez lateral de diseño. En las figs. 13a y 13b se observa que antes de que la estructura-A desarrolle el Vb-ELS su comportamiento es significativamente inelástico y por lo tanto no cumple los criterios de desempeño requeridos en el ELS. Por otra parte, el FAD puede interpretarse como la capacidad dúctil global total de deformación lateral, y sus valores obtenidos de las curvas de capacidad idealizadas como bilineales mostradas en las figs. $13 \mathrm{a}$ y $13 \mathrm{~b}$ son: $F A D-X=(\rho R=1.79)(Q=3.36)=5.99$ y $F A D$ $Y=(\rho R=1.62)(Q=3.85)=6.25$. Ambos valores son significativamente menores que los considerados en el diseño estructural: $F A D-X=F A D-Y=12.5$. Anteriormente se mencionó que la rigidez lateral elástica de la estructura considerada en el diseño estructural fue mayor que la obtenida en el análisis pushover, por lo que los efectos de segundo orden fueron más severos en éste análisis. Es conocido que los efectos de segundo orden reducen la sobrerresistencia y la capacidad de deformación lateral de una estructura, lo cual explica el resultado de que los $F A D$ obtenidos de la curva de capacidad fueron menores que los considerados en el diseño estructural. 


\section{Estructura-B}

Los periodos fundamentales elásticos traslacionales de esta estructura en ambas direcciones son: $T f x=1.403 \mathrm{~s}$ y $T f y=1.354 \mathrm{~s}$. Estos periodos son mayores en un $6 \%$ y $4 \%$, respectivamente, a los correspondientes periodos obtenidos del diseño estructural, $T f x=1.32 \mathrm{~s}$ y $T f y=1.30 \mathrm{~s}$. En la tabla 5 se puede observar que las relaciones $I_{\text {eff-prom }} / I_{g}$ de las vigas estimadas a partir de sus diagramas momento-curvatura, son significativamente mayores que dicha relación especificada por las NTC-DC-2017 para vigas, $I_{e f f} / I_{g}=0.5$; mientras que las relaciones $I_{\text {eff }} / I_{g}$ de columnas obtenidas de los diagramas son considerablemente menores que la especificada por dichas normas para columnas, $I_{\text {eff }} I_{g}=0.7$. Los resultados anteriores indican que el incremento en la rigidez de las vigas compensó en gran medida la disminución de la rigidez lateral de la estructura debida a decremento de la rigidez de las columnas. Debido a esto, la rigidez lateral elástica de la estructura decreció ligeramente y sus periodos se incrementaron en la misma proporción.

Del análisis pushover realizado a la estructura para cada dirección de análisis se obtuvieron las correspondientes curvas de capacidad mostradas en las figs. 14a y 14b. En estas figuras también se muestran los puntos definidos por el cortante basal y el correspondiente desplazamiento lateral del punto de la azotea mencionado anteriormente, en la correspondiente dirección de análisis; obtenidos de los análisis sísmicos elásticos de la estructura-B considerando el espectro de diseño correspondiente a cada estado límite. El hecho de que ambos puntos de respuesta estén fuera de la curva de capacidad se debe únicamente a que la rigidez lateral elástica de la estructura obtenida considerando la contribución del refuerzo longitudinal en los miembros, fue menor que la rigidez lateral de diseño, ya que en las figs. 14a y $14 \mathrm{~b}$ se observa que cuando la estructura-B alcanza el Vb-ELS; su respuesta es completamente elástica. Debido a lo anterior, esta estructura cumple los criterios de desempeño requeridos en el ELS. Por otra parte, los valores de los FAD obtenidos de las curvas de capacidad idealizadas como bilineales mostradas en las figs. 14a y 14b son: $F A D-X=(\rho R=1.42)(Q=2.38)=3.38$ y $F A D-Y=(\rho R=1.41)(Q=2.75)=3.89$. Ambos valores son menores que los considerados en el diseño estructural: $F A D-X=5.64$ y $F A D-Y=5.68$. La razón de este resultado es la misma que se discutió para la estructura-A.

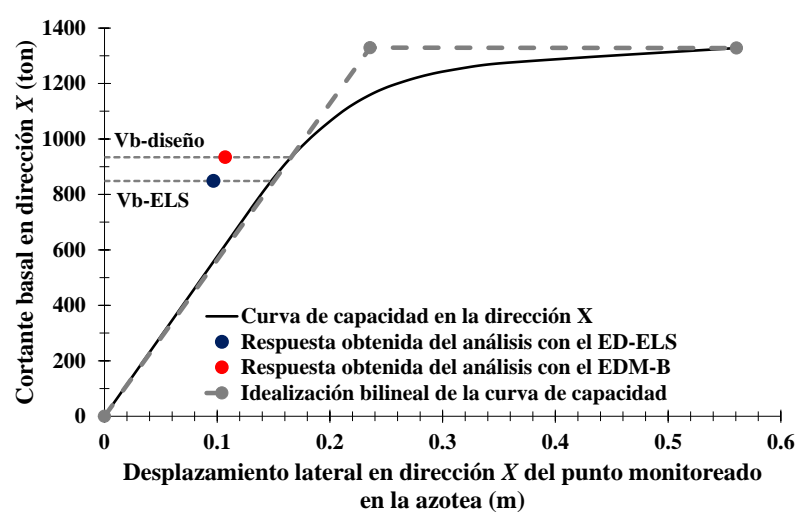

a) En la dirección $X$

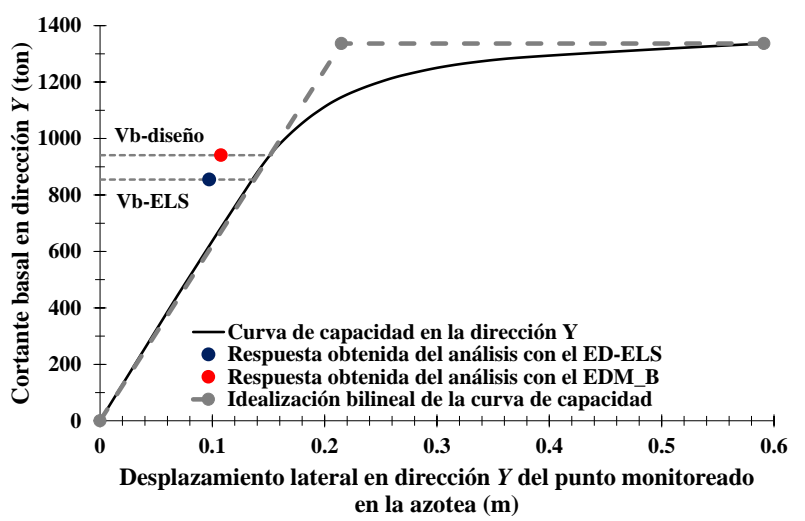

b) En la dirección $Y$

Figura 14. Curvas de capacidad en ambas direcciones de análisis de la estructura-B

\section{CONCLUSIONES Y RECOMENDACIONES}

Con base en los resultados mostrados en el presente trabajo se pueden realizar las siguientes conclusiones y recomendaciones, aplicables en principio, para estructuras tipo edificio con importancia estructural B1 en la región sísmica B. 
1. Se mostró que el diseñar estructuras tipo edificio cuyos periodos fundamentales traslacionales se ubiquen después de la rama ascendente de los espectros de diseño, de acuerdo a las recomendaciones del CDS-MDOC-2015 para un valor de su capacidad dúctil de deformación lateral, $Q$ igual a cuatro; puede resultar en que el estado límite de servicio rija el diseño por resistencia de una estructura, lo cual no debe ser. Para percatarse de ello, el diseñador tendrá que realizar un proceso iterativo que podría requerir muchas iteraciones, lo cual incrementará significativamente el trabajo. Por lo anterior, en el presente estudio se obtuvieron unas funciones para estimar el máximo valor de $Q$ con el que se debe diseñar un edificio, con cuya implementación se reduce considerablemente dicho trabajo iterativo y se cumplen simultáneamente los criterios de desempeño para ambos estados límite de diseño. Es importante tener presente que dichas funciones de $Q$ solo aplican cuando no se justifique tomar en cuenta los efectos de la interacción sueloestructura.

2. Los resultados obtenidos en el presente trabajo no implican que las estructuras cuyos periodos fundamentales traslacionales se ubiquen después de la rama ascendente de los espectros de diseño, no deban diseñarse para proporcionarles una capacidad dúctil global de deformación lateral de cuatro. De hecho, en la medida que el proyecto arquitectónico lo permita, es recomendable proponer un sistema estructural con materiales cuya naturaleza no sea frágil, que tengan una configuración geométrica regular con la suficiente redundancia estructural, así como realizar un "detallado sísmico" adecuado de sus miembros y las conexiones entre ellos; que permitan proporcionarle la mayor capacidad dúctil global de deformación lateral posible.

3. Es recomendable realizar un estudio para definir el periodo de retorno óptimo de diseño para el estado límite de servicio de estructuras tipo edificio ubicadas en el municipio de Veracruz. Con base en este periodo de retorno, puede calcularse el espectro de diseño para dicho estado límite y el correspondiente $F_{\text {ser }}$.

\section{AGRADECIMIENTOS}

El primer autor agradece a la Secretaría de Educación Pública el apoyo económico otorgado para realizar el presente trabajo, a través del Programa para el Desarrollo Profesional Docente para el Tipo Superior. También se agradecen las observaciones y sugerencias de los revisores anónimos de este documento.

\section{REFERENCIAS}

CDS-MDOC-CFE (2015), Capítulo de diseño por sismo. Manual de diseño de obras civiles, versión 2015, Comisión Federal de Electricidad, México.

Corona, G (2017), ECOgcW3, programa para análisis y diseño de edificios, Guía rápida de uso, www.gcingenieria.com

Elnashai, A S, y A M Mwafy, (2002), "Overstrenght and force reduction factors of multistory reinforcedconcrete buildings", The structural design of tall buildings, Vol. 11, pp. 329-351. DOI: $\underline{10.1002 / \mathrm{tal} .204}$

NTC-DC (2017), Normas técnicas complementarias para diseño y construcción de estructuras de concreto, Reglamento de construcciones de la Ciudad de México, Gaceta oficial de la Ciudad de México, México. 
NTC-CA (2017), Normas técnicas complementarias sobre criterios y acciones para el diseño estructural de las edificaciones, Reglamento de construcciones de la Ciudad de México, Gaceta oficial de la Ciudad de México, México.

SAP2000 v.19.2 (2017), Integrated finite element analysis and design of structures, Computer and Structures Inc, Berkeley, California, Estados Unidos.

Rodríguez, M, y J C Botero, (1996), "Aspectos del comportamiento sísmico de estructuras de concreto reforzado considerando las propiedades mecánicas de aceros de refuerzo producidos en México", Series del Instituto de Ingeniería, UNAM, 575.

Mander, J B, M J N Priestley y R Park, (1988), "Theorical stress-strain model for confined concrete", Journal of Structural Engineering, ASCE, Vol. 33, No. 8, pp. 1804-1826. DOI: 10.1061/(ASCE)0733-9445(1988)114:8(1804)

Uang, C, (1991), "Establishing $\mathrm{R}$ (or $\mathrm{R}_{\mathrm{w}}$ ) and $\mathrm{C}_{\mathrm{d}}$ factors for buildings seismic provisions", Journal of Structural Engineering, ASCE, Vol. 117, No. 1, pp. 19-28. DOI: 10.1061/(ASCE)07339445(1991)117:1(19) 